\title{
The effect of two distinct fast time scales in the rotating, stratified Boussinesq equations: Variations from Quasi-Geostrophy
}

\author{
Jared P. Whitehead ${ }^{1}$. Terry Haut ${ }^{2}$ - Beth
}

A. Wingate ${ }^{3}$

the date of receipt and acceptance should be inserted later

\begin{abstract}
Inspired by the use of fast singular limits in time-parallel numerical methods for a single fast frequency, we consider the limiting, nonlinear dynamics for a system of partial differential equations when two fast, distinct time scales are present. First order slow equations are derived via the method of multiple time scales when the two small parameters are related by a rational power. We find that the resultant system depends only on the relationship of the two fast time-scales, i.e. which fast time is fastest? Using the theory of cancellation of fast oscillations, we show that with the appropriate assumptions on the nonlinear operator of the full system, this reduced slow system is exactly that which the solution will converge to if each asymptotic limit is considered sequentially. The same result is also obtained via the method of renormalization. The specific example of the rotating, stratified Boussinesq equations is explored in detail, indicating that the most common distinguished limit of this system - quasi-geostrophy, is not the only limiting asymptotic system.
\end{abstract}

\section{Introduction}

The evolution of physical systems on disparate time scales is frequently observed in many branches of the physical sciences including the dynamics of the atmosphere and oceans that will serve as the main example in this paper [6,7]. Rapid oscillatory components of these systems lead to significant numerical stiffness due to severe restrictions on the time step required to maintain accuracy. This stiffness

J.P. Whitehead

Department of Mathematics, Brigham Young University, Provo, UT, USA 84602, E-mail: whitehead@math.byu.edu

T. Haut

Lawrence Livermore National Laboratory, Center for Applied Scientific Computing, P. O. Box 8080, L-561 Livermore, CA 94551 E-mail: haut3@llnl.gov

B.A. Wingate

Department of Mathematics, University of Exeter, Exeter, UK EX4 4QF, E-mail: b.wingate@exeter.ac.uk 
reduces the predictive capability of any mathematical model of the physical system. One approach to overcoming these obstacles is the implementation of time parallel numerical methods such as the parareal algorithm developed by Lions, Maday and Turinici $[25]$ (see $[18,17]$ for a review of time-parallel methods). Quantifying the numerical properties of time-parallel algorithms, especially when the stiffness is due to oscillations, is a challenging task but recent numerical advances motivated by the mathematics that underpin fast singular limits have successfully designed time-parallel numerical schemes that have superlinear convergence in the limit as the time-scale separation goes to infinity and when the linear operator is skew-Hermitian [20]. This result relies on the fact that there is a single fast frequency in the system even though many physical systems have more than one (for example, rotation and buoyancy). Investigation of fast singular limits of PDEs that have more than one fast frequency can then lead to more feasible numerical implementations, particularly in terms of time-parallel methods.

The reason [20] could develop parallel speedup even for problems with oscillatory stiffness in the governing equations is because they made use of the mathematics underpinning separation of time scales from fast singular limits. That is, rapid oscillations lead to a separation of temporal scales that yield asymptotically derived reduced equations that govern the slow dynamics of the system. These slow equations have the practical advantage of being more efficient to integrate numerically. A geophysical example of this is the first successful numerical weather forecast developed by Charney (see $[7,8]$ ). To remove the inherent stiffness in weather prediction, [7] deduced the slow equations by comparing the magnitude of terms in the full system via atmospheric weather data and omitting those terms of significantly smaller relative magnitude. Since the remarkable success of Charney's quasi-geostrophic (QG) approximation, slow equations (sometimes QG, and sometimes distinct from QG) have been derived in a variety of other ways (see $[30,4,9,13,14,21,32,37,22]$ for some examples). For more additional formal accuracy, corrections to the equations in the form of higher order expansions have been proposed (see $[26,42,29,39,40,32]$ for some examples as well as [22] where spatial anisotropy is considered a factor as well).

Because the asymptotic reductions in this context are typically singular, the path taken to the limiting system matters, i.e. taking two asymptotic limits consecutively is generically not commutative. Partially for this reason, we will focus on asymptotically motivated reductions from a system that includes two fast time scales (represented by two vanishing parameters $\delta$ and $\epsilon$ ) but does not assume equivalency of the fast scales $(\delta / \epsilon \approx O(1)$, as is done to derive QG). Although the primary motivation for this derivation arises in fluid dynamics, it is presented in a general formulation as the methodology is quite general. Other than adequate restrictions on the strength of the nonlinearity of the system, there are two primary requirements: 1) the two fast time scales are dictated by skew-Hermitian linear operators and 2) the spectrum of the fast operators is discrete, typically meaning that the spatial domain of consideration is bounded. Such considerations are the same as those relied on in $[13,14]$ for geophysical applications.

We analyze the singular limit of two asymptotically limiting skew-Hermitian operators which function on two distinct time scales. This is distinct from [12] and [5] where two distinct fast time-scales are present, but the relation between the scales is specified i.e. $\delta=\epsilon^{2}$ below, effectively reducing the problem to a single fast time scale. Further consideration of the low Mach number and compressible 
limits do consider multiple fast time scales [35]. [35] provides a clear overview of the use of multi-scale asymptotics in such a setting, but also introduces additional spatial scales which then provide a restriction on the domain of interest and/or the initial conditions of the problem at hand. The current investigation considers various scales in time only, eliminating restrictions/conditions on the domain but also relying on the need to consider either the whole space or a periodic domain. [16] looks into a limiting situation very similar to that presented here, but for compressible fluids which requires a very different methodology based on relative entropies rather than the use of energy estimates. From a fundamental perspective the current result applies the method of multiple scales in a general setting to situations where energy-type estimates are applicable and there are two fast scales whose inter-relationship is unknown other than relative speed to each other.

The current investigation is of particular interest in a geophysical context when considered in light of the result of [41] that indicates that QG dominates the solution for sufficiently long times for solutions of the hydrostatic primitive equations. This relies on the fact that for this system (as in the case of the Boussinesq system considered in $[14,43]$ ) and the corresponding asymptotic reduction, the influence of the fast waves on the slow dynamics vanishes to $O(1)$. We show that a similar condition holds for the reduced dynamics of the Boussinesq system when two distinct fast time scales are considered.

Using the method of cancellation of oscillations (see $[23,34]$ ) we show that the reduced systems obtained via multiple scales, are verifiably the limit of the full system if the limits are taken sequentially. In other words, if $\delta \ll \epsilon$ then the multiple scales derivation leads to a reduced slow system that is rigorously given by taking the limit $\delta \rightarrow 0$ first, and then letting $\epsilon \rightarrow 0$. In the context of the Boussinesq system, the secondary limit (slower fast scale as given by $\epsilon$ in this example) only affects spatially averaged quantities of the flow.

Though the PDE examples in this paper are from geophysical fluid dynamics, the approach outlined in Section 2 is generally applicable to any system (ordinary or partial differential equation) with two skew-Hermitian linear operators with a discrete spectrum, and a quadratic nonlinear term (provided sufficient conditions are placed on the strength of the nonlinearity, such as that discussed in [13]). As mentioned previously, the asymptotic reductions performed here are of interest in the context of time-parallel methods (see $[15,2,20]$ and citations therein as well as [1] where three distinct time scales motivate a particular time-stepping algorithm).

The next section contains the derivation of the limiting set of equations for a generic system of differential equations (partial or ordinary) for which there are two skew-Hermitian linear operators where the corresponding small parameters are related as an integer power greater than 2. Section 3 extends this result to include the more general case of all pairs of small parameters such that they are related via a rational exponent. Section 4 considers this same problem in the context of the methods of cancellation of oscillations (see [34] for the primary motivation) and the renormalization group method, providing a brief summary of the insights gained from each of the three approaches. Section 5 introduces the rotating stratified Boussinesq equations and investigates the two possible limits of rapid rotation dominating strong stratification and vice versa in the context of the reduced dynamics derived in Section 2. We conclude with some brief discussion in Section 6, and an Appendix detailing one of the more mundane calculations. 


\section{Separation of three time-scales}

We consider the system of equations governed by

$$
\frac{\partial \boldsymbol{u}}{\partial t}+\frac{1}{\delta} \mathcal{L} \boldsymbol{u}+\frac{1}{\epsilon} \mathcal{K} \boldsymbol{u}+\mathcal{B}(\boldsymbol{u}, \boldsymbol{u})=\mathcal{D} \boldsymbol{u}
$$

where $\boldsymbol{u}=\boldsymbol{u}(t, \boldsymbol{x})$ is a vector valued function of time (and possibly space), and the linear operators $\mathcal{L}$ and $\mathcal{K}$ are skew-Hermitian. The nonlinearity of the equation is contained in $B(\boldsymbol{u}, \boldsymbol{u})$, and is inherently quadratic (although extensions to higher order polynomial nonlinearities are possible), and the dissipative operator $\mathcal{D}$ does not directly affect the following, but is included for completeness. Boundary conditions are important in all that follows, particularly in the sense that $\mathcal{L}$ and $\mathcal{K}$ must have a discrete spectrum, but the precise specification of the boundary is omitted for complete generality. The goal here is to consider the simultaneous singular limits $\epsilon, \delta \rightarrow 0$ in the case that $\delta \ll \epsilon$, i.e. both $\mathcal{L}$ and $\mathcal{K}$ create 'fast' oscillations, but the motion induced by $\mathcal{L}$ is 'faster' than that induced by $\mathcal{K}$. In this Section we will assume that the small parameters $\epsilon$ and $\delta$ are related as $\delta=\epsilon^{p}$ where $p \in \mathbb{Z}_{+}$ and $p>2$. The extension of these results to the distinguishing cases of $p=2$ and for $p$ rational are considered in the following Section.

We consider the formalism of multiple scale asymptotics in detail (see $[13,14$, $43]$ ) and briefly discuss a similar derivation via the renormalization group method (see $[44,31]$ ). The focus on the formalism of multiple scales is due to the simplicity of the calculation that follows. Although the final result is the same via renormalization, the calculation is far more complicated, and only a specific example is given in Section 4.

We consider the evolution of the solution $\boldsymbol{u}$ to (1) in a perturbative approach in which three separate time scales, $t, \tau=t / \epsilon$, and $\alpha=t / \delta$ are present. For $\epsilon \ll 1$ (and correspondingly $\delta \ll 1$ ) we enforce the ansatz

$$
\boldsymbol{u}(t, \boldsymbol{x})=\boldsymbol{u}_{0}(t, \tau, \alpha, \boldsymbol{x})+\epsilon \boldsymbol{u}_{1}(t, \tau, \alpha, \boldsymbol{x})+O\left(\epsilon^{2}\right) .
$$

This yields for the highest order $O\left(\frac{1}{\epsilon^{p}}\right)$ term:

$$
\frac{\partial \boldsymbol{u}_{0}}{\partial \alpha}+\mathcal{L} \boldsymbol{u}_{0}=0
$$

which has solution

$$
\boldsymbol{u}_{0}(t, \tau, \alpha, \boldsymbol{x})=e^{-\alpha \mathcal{L}} \hat{\boldsymbol{u}}_{0}(t, \tau, \boldsymbol{x}),
$$

where evaluation of $e^{-\alpha \mathcal{L}}$ is dependent on the boundary conditions and geometry of the underlying problem and $\hat{\boldsymbol{u}}_{0}$ is independent of $\alpha$.

The next $p-2$ terms (given by $O\left(\frac{1}{\epsilon^{q}}\right)$ for $q=p-1, p-2, \ldots 2$ ) are

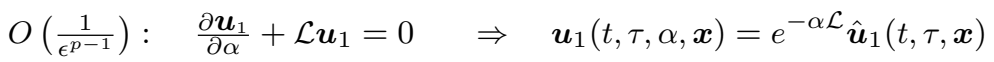

$$
\begin{aligned}
& \vdots \quad \vdots \\
& O\left(\frac{1}{\epsilon^{2}}\right): \quad \frac{\partial \boldsymbol{u}_{p-2}}{\partial \alpha}+\mathcal{L} \boldsymbol{u}_{p-2}=0 \quad \Rightarrow \quad \boldsymbol{u}_{p-2}(t, \tau, \alpha, \boldsymbol{x})=e^{-\alpha \mathcal{L}} \hat{\boldsymbol{u}}_{p-2}(t, \tau, \boldsymbol{x})
\end{aligned}
$$


and the final two pertinent terms in the expansion are

$$
\begin{array}{ll}
O\left(\frac{1}{\epsilon}\right): & \frac{\partial \boldsymbol{u}_{p-1}}{\partial \alpha}+\mathcal{L} \boldsymbol{u}_{p-1}+\frac{\partial \boldsymbol{u}_{0}}{\partial \tau}+\mathcal{K} \boldsymbol{u}_{0}=0 \\
O(1): & \frac{\partial \boldsymbol{u}_{p}}{\partial \alpha}+\mathcal{L} \boldsymbol{u}_{p}+\frac{\partial \boldsymbol{u}_{1}}{\partial \tau}+\mathcal{K} \boldsymbol{u}_{1}+\frac{\partial \boldsymbol{u}_{0}}{\partial t}+\mathcal{B}\left(\boldsymbol{u}_{0}, \boldsymbol{u}_{0}\right)-\mathcal{D} \boldsymbol{u}_{0}=0 .
\end{array}
$$

Solving (6), and using (4) leads to

$$
e^{\alpha \mathcal{L}} \boldsymbol{u}_{p-1}=\left.\boldsymbol{u}_{p-1}\right|_{\alpha=0}-\alpha \frac{\partial \hat{\boldsymbol{u}}_{0}}{\partial \tau}-\left(\int_{0}^{\alpha} e^{s \mathcal{L}} \mathcal{K} e^{-s \mathcal{L}} d s\right) \hat{\boldsymbol{u}}_{0}
$$

Noting that the right hand side of the above (except the first term) clearly has dependence on $\alpha$, potentially leading to secular growth of $\boldsymbol{u}_{p-1}$ we enforce the following sufficient condition to eliminate secularity:

$$
\alpha \frac{\partial \hat{\boldsymbol{u}}_{0}}{\partial \tau}+\left(\int_{0}^{\alpha} e^{s \mathcal{L}} \mathcal{K} e^{-s \mathcal{L}} d s\right) \hat{\boldsymbol{u}}_{0}=0,
$$

preferably for $\alpha$ as large as possible. With this in mind, we arrive at the following statement to avoid secular growth of $\boldsymbol{u}_{p-1}$ :

$$
\frac{\partial \hat{\boldsymbol{u}}_{0}}{\partial \tau}=-\left(\lim _{\alpha \rightarrow \infty} \frac{1}{\alpha} \int_{0}^{\alpha} e^{s \mathcal{L}} \mathcal{K} e^{-s \mathcal{L}} d s\right) \hat{\boldsymbol{u}}_{0}
$$

which can be solved directly (where the details of these operators again depend on the boundary conditions and geometry):

$$
\hat{\boldsymbol{u}}_{0}(t, \tau, \boldsymbol{x})=e^{-\tau \overline{\mathcal{M}}} \overline{\boldsymbol{u}}_{0}(t, \boldsymbol{x}) \quad \Rightarrow \quad \boldsymbol{u}_{0}(t, \tau, \alpha, \boldsymbol{x})=e^{-\tau \mathcal{L}} e^{-\alpha \overline{\mathcal{M}}} \overline{\boldsymbol{u}}_{0}(t, \boldsymbol{x})
$$

where the linear operator $\mathcal{M}(\alpha)$ is given by

$$
\mathcal{M}(\alpha)=\frac{1}{\alpha} \int_{0}^{\alpha} e^{s \mathcal{L}} \mathcal{K} e^{-s \mathcal{L}} d s
$$

and $\overline{\mathcal{M}}=\lim _{\alpha \rightarrow \infty} \mathcal{M}(\alpha)$. Hence, returning to (8) we can write

$$
\boldsymbol{u}_{p-1}(t, \tau, \alpha, \boldsymbol{x})=e^{-\alpha \mathcal{L}} \hat{\boldsymbol{u}}_{p-1}(t, \tau, \boldsymbol{x})+\alpha e^{-\alpha \mathcal{L}}(\overline{\mathcal{M}}-\mathcal{M}(\alpha)) e^{-\tau \overline{\mathcal{M}}} \overline{\boldsymbol{u}}_{0}(t, \boldsymbol{x}) .
$$

Although this appears to yield secular growth in $\alpha$ for $\boldsymbol{u}_{p-1}$, in reality $\alpha(\overline{\mathcal{M}}-\mathcal{M}(\alpha))=$ $O(1)$ and is bounded (as shown explicitly in Section 3.1). Hence in the limit of complete separation of scales or $\epsilon \rightarrow 0$ this will not effect the slowly evolving dynamics.

In a similar fashion we can solve for the $\alpha$ dependence of (7) as

$$
\begin{aligned}
e^{\alpha \mathcal{L}} \boldsymbol{u}_{p} & =\hat{\boldsymbol{u}}_{p}-\alpha \frac{\partial \hat{\boldsymbol{u}_{1}}}{\partial \tau}-\alpha \mathcal{M}(\alpha) \hat{\boldsymbol{u}}_{1}-\left(\int_{0}^{\alpha} e^{s \mathcal{L}} e^{-\tau \overline{\mathcal{M}}} e^{-s \mathcal{L}} d s\right) \frac{\partial \overline{\boldsymbol{u}}_{0}}{\partial t} \\
& -\int_{0}^{\alpha} e^{s \mathcal{L}} \mathcal{B}\left(e^{-\tau \overline{\mathcal{M}}} e^{-s \mathcal{L}} \overline{\boldsymbol{u}}_{0}, e^{-\tau \overline{\mathcal{M}}} e^{-s \mathcal{L}} \overline{\boldsymbol{u}}_{0}\right) d s+\left(\int_{0}^{\alpha} e^{s \mathcal{L}} \mathcal{D} e^{-\tau \overline{\mathcal{M}}} e^{-s \mathcal{L}} d s\right) \overline{\boldsymbol{u}}_{0},
\end{aligned}
$$


which, to avoid secularity in $\alpha$ (in the limit as $\alpha \rightarrow \infty$ ), leads to the $\tau$ dependence of $\boldsymbol{u}_{1}$ as

$$
\begin{aligned}
e^{\tau \mathcal{M}} \hat{\boldsymbol{u}}_{1} & =\overline{\boldsymbol{u}}_{1}-\mathcal{N}(\tau) \frac{\partial \overline{\boldsymbol{u}}_{0}}{\partial t} \\
& -\int_{0}^{\tau} e^{\beta \overline{\mathcal{M}}}\left[\lim _{\alpha \rightarrow \infty} \frac{1}{\alpha} \int_{0}^{\alpha} e^{\alpha \mathcal{L}} \mathcal{B}\left(e^{-\beta \overline{\mathcal{M}}} e^{-s \mathcal{L}} \overline{\boldsymbol{u}}_{0}, e^{-\beta \overline{\mathcal{M}}} e^{-s \mathcal{L}} \overline{\boldsymbol{u}}_{0}\right) d s\right] d \beta(15) \\
& +\left(\int_{0}^{\tau} e^{\beta \overline{\mathcal{M}}}\left[\lim _{\alpha \rightarrow \infty} \frac{1}{\alpha} \int_{0}^{\alpha} e^{s \mathcal{L}} \mathcal{D} e^{-\beta \overline{\mathcal{M}}} e^{-s \mathcal{L}} d s\right] d \beta\right) \overline{\boldsymbol{u}}_{0}
\end{aligned}
$$

where

$$
\mathcal{N}(\tau)=\lim _{\alpha \rightarrow \infty} \frac{1}{\alpha} \int_{0}^{\tau} \int_{0}^{\alpha}\left(e^{\beta \overline{\mathcal{M}}} e^{s \mathcal{L}} e^{-\beta \overline{\mathcal{M}}} e^{-s \mathcal{L}}\right) d s d \beta
$$

Avoiding secular growth of $\hat{\boldsymbol{u}}_{1}$ as $\tau \rightarrow \infty$, and noting that since $\mathcal{L}$ and $\overline{\mathcal{M}}$ commute (when $\mathcal{L}$ and $\mathcal{K}$ both have a discrete spectrum, see Appendix A) then $\mathcal{N}(\tau)=\tau$, leads to the slow $O(1)$ evolution equation:

$$
\frac{\partial \overline{\boldsymbol{u}}_{0}}{\partial t}=-\lim _{\tau \rightarrow \infty} \lim _{\alpha \rightarrow \infty} \frac{1}{\tau \alpha} \int_{0}^{\tau} \int_{0}^{\alpha} e^{\beta \overline{\mathcal{M}}} e^{s \mathcal{L}} \mathcal{B}\left(e^{-\beta \overline{\mathcal{M}}} e^{-s \mathcal{L}} \overline{\boldsymbol{u}}_{0}, e^{-\beta \overline{\mathcal{M}}} e^{-s \mathcal{L}} \overline{\boldsymbol{u}}_{0}\right) d s d \beta+D \overline{\boldsymbol{u}}_{0}
$$

where the linear dissipative operator $D$ is defined

$$
D=\lim _{\tau \rightarrow \infty} \lim _{\alpha \rightarrow \infty} \frac{1}{\tau \alpha} \int_{0}^{\tau} \int_{0}^{\alpha} e^{\beta \overline{\mathcal{M}}} e^{s \mathcal{L}} \mathcal{D} e^{-\beta \overline{\mathcal{M}}} e^{-s \mathcal{L}} d s d \beta
$$

The derivation of (17) does not rely on the exact value of $p$, only on which scale is 'fastest' (which of $\delta$ and $\epsilon$ approaches zero the fastest), although as noted in the next Section, the derivation is more complicated when $p=2$, and for some rational powers. This provides a generic result that yields a surprisingly simple formula for the first order slow dynamics (see $[14,43]$ for some other examples) that is more widely applicable than the case $\delta=O(\epsilon)$ (considered for the quasi-geostrophic limit of the Boussinesq system [14]). Significantly, the 'fast' dynamics depends more strongly on the fastest linear operator $\mathcal{L}$, and only indirectly on the other 'fast' operator $\mathcal{K}$. However, the $O(1)$ dynamics as derived here are valid only up to corrections whose magnitude is the order of the larger of the small parameters, $\epsilon$. In this sense, the 'fastest' operator dictates the behavior of the $O(1)$ limiting slow dynamics, but the slower of the fast operators dictates the accuracy of the approximation.

\section{Generic extensions from integer powers}

The results of the previous Section yield a powerful description of the asymptotic behavior of the doubly singular limit described by (1). These results can be extended further than assuming an integer power (greater than 2) relationship between the small parameters $\epsilon$ and $\delta$ however. This derivation is depicted for ease of presentation, and the next two subsections demonstrate some extensions of this primary result to generalize the applicability of the reduced system in (17). 
3.1 Quadratically related small parameters

Suppose that $p=2$ in the previous section. This indicates that the relevant equations in the expansion are (3) (here $O\left(\frac{1}{\epsilon^{2}}\right)$ ), and $\boldsymbol{u}_{p-1} \rightarrow \boldsymbol{u}_{1}$ and $\boldsymbol{u}_{p} \rightarrow \boldsymbol{u}_{2}$ in (6), (7). This indicates that the same secularity condition is applied to (6) (with respect to $\alpha$ ) to restrict secular growth of $\boldsymbol{u}_{1}$ in $\alpha$, once again leading to (11). (13) then holds for $\boldsymbol{u}_{1}$, and we can solve for the $\alpha$ dependence of $\boldsymbol{u}_{2}$ in (7) as (after integrating by parts appropriately):

$$
\begin{aligned}
e^{\alpha \mathcal{L}} \boldsymbol{u}_{2} & =\hat{\boldsymbol{u}}_{2}-\alpha \frac{\partial \hat{\boldsymbol{u}}_{1}}{\partial \tau}-\alpha \mathcal{M}(\alpha) \hat{\boldsymbol{u}}_{1}+\alpha^{2}\left[\frac{1}{2} \overline{\mathcal{M}}^{2}-\overline{\mathcal{M}} \mathcal{M}(\alpha)+\frac{1}{2} \mathcal{M}(\alpha)^{2}\right] e^{-\tau \overline{\mathcal{M}}} \overline{\boldsymbol{u}}_{0} \\
& -\alpha e^{-\tau \overline{\mathcal{M}}} \frac{\partial \overline{\boldsymbol{u}}_{0}}{\partial t}-\int_{0}^{\alpha} e^{s \mathcal{L}} \mathcal{B}\left(e^{-\tau \overline{\mathcal{M}}} e^{-s \mathcal{L}} \overline{\boldsymbol{u}}_{0}, e^{-\tau \overline{\mathcal{M}}} e^{-s \mathcal{L}} \overline{\boldsymbol{u}}_{0}\right) d s \\
& +\left(\int_{0}^{\alpha} e^{s \mathcal{L}} \mathcal{D} e^{-s \mathcal{L}} d s\right) e^{-\tau \overline{\mathcal{M}}} \overline{\boldsymbol{u}}_{0}
\end{aligned}
$$

In order to ensure there is no secular growth of $\boldsymbol{u}_{2}$ in $\alpha$ we force all of the terms from the above involving $\boldsymbol{u}_{1}$ and $\boldsymbol{u}_{0}$ to vanish in the limit as $\alpha \rightarrow \infty$. Conveniently (and in concert with the ansatz used in this asymptotic expansion), all the terms involving $\alpha^{2}$ above cancel as $\alpha \rightarrow \infty$. To see this we rewrite the integral operator $\mathcal{M}(\alpha)$ as a truncated series about $\alpha=\infty$ :

$$
\mathcal{M}(\alpha)=\overline{\mathcal{M}}+\frac{\mathcal{V}(\alpha)}{\alpha}
$$

where $\mathcal{V}(\alpha)$ may oscillate very rapidly, but remains $O(1)$ (bounded in fact) for all $\alpha$. With this substitution the nearly secular term in (19) is given by

$$
\alpha^{2}\left[\frac{1}{2} \overline{\mathcal{M}}^{2}-\overline{\mathcal{M}} \mathcal{M}(\alpha)+\frac{1}{2} \mathcal{M}(\alpha)^{2}\right]=\frac{1}{2} \mathcal{V}(\alpha)^{2},
$$

which will be rapidly oscillatory for large $\alpha$, but remains bounded.

Noting that this near secular term is in fact $O(1)$ in $\alpha$ leads to the solution of $\boldsymbol{u}_{1}$ in terms of $\tau$ as given by (15), and eventually to the final solution of the $O(1)$ 'slow' dynamics given by (17). Hence, as noted in the previous Section the $O(1)$ slow dynamics are not directly affected by this near secularity, but the following two higher order terms in the expansion will have rapid oscillatory (non-convergent) coupling with the $O(1)$ slow dynamics.

\subsection{Rationally related small parameters}

Consider the case of $\delta=\epsilon^{\frac{p}{q}}$ where $p, q \in \mathbb{Z}_{+}$, i.e. the two small parameters of (1) are related by by a rational power. In this case we consider the transformation $\epsilon \rightarrow \epsilon^{q}$ so that (1) becomes

$$
\frac{\partial \boldsymbol{u}}{\partial t}+\frac{1}{\epsilon^{p}} \mathcal{L} \boldsymbol{u}+\frac{1}{\epsilon^{q}} \mathcal{K} \boldsymbol{u}+\mathcal{B}(\boldsymbol{u}, \boldsymbol{u})=\mathcal{D} \boldsymbol{u} .
$$

Without loss of generality we assume that $p>q+1$ (the case of $p=q+1$ leads to additional near secular terms akin to those discussed in the previous subsection, 
coupling the $O(1)$ slow dynamics to the next $q+1$ terms in the expansion through a rapidly non-convergent oscillatory function of $\alpha$ ) so that once again $\mathcal{L}$ is the dominant operator. We then consider the asymptotic expansion now with time scales $\tau=\frac{t}{\epsilon^{q}}$ and $\alpha=\frac{t}{\epsilon^{p}}$ combined with

$$
\boldsymbol{u}(t, \boldsymbol{x})=\boldsymbol{u}_{0}(t, \tau, \alpha, \boldsymbol{x})+\epsilon^{q} \boldsymbol{u}_{q}(t, \tau, \alpha, \boldsymbol{x})+O\left(\epsilon^{q+1}\right) .
$$

This leads to the same development as in the previous Section with the highest order term indicating that (4) holds in this case, and (6) being replaced by

$$
O\left(\frac{1}{\epsilon^{q}}\right): \quad \frac{\partial \boldsymbol{u}_{p-q}}{\partial \alpha}+\mathcal{L} \boldsymbol{u}_{p-q}+\frac{\partial \boldsymbol{u}_{0}}{\partial \tau}+\mathcal{K} \boldsymbol{u}_{0}=0
$$

also implying that (11) is sufficient to avoid secular growth of $\boldsymbol{u}_{p-q}$ with $\alpha$. (7) is replaced by:

$$
O(1): \quad \frac{\partial \boldsymbol{u}_{p}}{\partial \alpha}+\mathcal{L} \boldsymbol{u}_{p}+\frac{\partial \boldsymbol{u}_{q}}{\partial \tau}+\mathcal{K} \boldsymbol{u}_{q}+\frac{\partial \boldsymbol{u}_{0}}{\partial t}+\mathcal{B}\left(\boldsymbol{u}_{0}, \boldsymbol{u}_{0}\right)-\mathcal{D} \boldsymbol{u}_{0}=0,
$$

where avoidance of secular growth of $\boldsymbol{u}_{p}$ in $\alpha$ and $\boldsymbol{u}_{q}$ in $\tau$ (the dependence of $\boldsymbol{u}_{q}$ on $\alpha$ is given by the $O\left(\frac{1}{\epsilon^{p-q}}\right)$ term) requires that (17) is satisfied.

This derivation is even more robust however, due to the density of rational numbers on the real number line, we can extrapolate these results to include any relation between the two small parameters. That is, as the two small parameters vanish, if there is a functional relationship that holds as the limit is approached then the slow $O(1)$ equations are identical to those given in (17) where the only important aspect of the functional relationship between $\epsilon$ and $\delta$ is that it is monotonic.

\section{Cancellation of oscillations and renormalization}

The previous two Sections considered the effect of two rapid, 'wave-generating' linear operators using the formalism of multiple time scale asymptotics. In the current Section we derive the same limiting system (with less attention paid to detail), but here using the methods of cancellation of fast oscillation (see $[3,23,34$, $19])$ and renormalization $([44,39,31,40])$. Although the same result is achieved for each of these approaches, the outline of each is included here to demonstrate the insight gained via each method. We will first discuss the method of cancellation of oscillations based primarily on the seminal paper by Schochet (see [34]), then consider a particular case for the renormalization group method before briefly highlighting the additional information each of these methods provides toward understanding the underlying physical system.

\subsection{Cancellation of rapid oscillations}

As noted in [13], the method of multiple scales provides motivation for the theory of cancellation of oscillations as established by [3,23,34,19]. Following [13] (see the beginning of their Section 3), we assume the following for the system (1): 
- Let $s>\frac{d}{2}+1$ and $X_{s}$ be a subset of $H^{s}\left(T^{d}\right)$ where $T^{d}$ is the $d$-dimensional periodic space. Then solutions of (1) with initial data $\boldsymbol{u}_{0}(\boldsymbol{x}) \in X_{s}$ will also lie in $X_{s}$ for $t \in[0, T]$ for a $T$ independent of $\epsilon$ and $\delta$, and

$$
\max \|\boldsymbol{u}(\boldsymbol{x}, t)\|_{s} \leq C\left(T,\left\|\boldsymbol{u}_{0}(\boldsymbol{x})\right\|_{H^{s}}\right)
$$

where the maximum is taken over the interval $[0, T]$ and for all $\epsilon$ and $\delta$ less than unity.

- As already established, we are concerned with cases for which $\mathcal{L}$ and $\mathcal{K}$ are skew-Hermitian with a discrete spectrum in which case their exponential is norm preserving and has purely imaginary, discrete spectrum.

As noted in [13] these assumptions allow us to extend the theory of [34] to the nonlinear system (1). We note here that as shown in [13] these assumptions hold for the Boussinesq system considered in Section 5.

Of particular interest, we note that the first assumption is necessary to adequately control terms arising as 'small divisors' or near resonances, i.e. for the Boussinesq system explicitly considered in this work a spectral gap between the slow and fast parts of the solution is violated for very high wave numbers. The first assumption listed above indicates that such terms in a Galerkin type expansion may be significant, but will always be bounded, i.e. infinitely small scales are not possible, and hence the necessity of a spectral gap is lessened. The cost of this assumption is that the coefficient in front of the higher order term may be quite large (although remaining bounded) so that the error terms are no longer $O(\epsilon)$, but $o(\epsilon)$ instead.

To see how the theory of cancellation of oscillations applies in this case, we will briefly demonstrate how the methodology of [34] can be applied in this case. In the current notation (and following that employed in [13]), and supposing that $\delta \ll \epsilon$ we consider first the change of variables

$$
\hat{\boldsymbol{u}}(t, \alpha, \boldsymbol{x})=e^{\left(\alpha-\frac{t}{\delta}\right) \mathcal{L}} \boldsymbol{u}(t, \boldsymbol{x})
$$

where $\boldsymbol{u}(t, \boldsymbol{x})$ is the solution of (1) with appropriately prescribed initial data. Not only are $\hat{\boldsymbol{u}}$ and $\frac{\partial \hat{\boldsymbol{u}}}{\partial t}$ bounded, but as evidenced by [13] (and outlined carefully in [34]), $\hat{\boldsymbol{u}}$ satisfies

$$
\begin{aligned}
& \frac{\partial \hat{\boldsymbol{u}}}{\partial t}+\frac{1}{\epsilon} \overline{\mathcal{M}} \hat{\boldsymbol{u}}+\lim _{\alpha \rightarrow \infty} \frac{1}{\alpha} \int_{0}^{\alpha} e^{s \mathcal{L}} \mathcal{B}\left(e^{-s \mathcal{L}} \hat{\boldsymbol{u}}, e^{-s \mathcal{L}} \hat{\boldsymbol{u}}\right) d s \\
= & \left(\lim _{\alpha \rightarrow \infty} \frac{1}{\alpha} \int_{0}^{\alpha} e^{s \mathcal{L}} \mathcal{D} e^{-s \mathcal{L}} d s\right) \hat{\boldsymbol{u}},
\end{aligned}
$$

in the limit $\delta \rightarrow 0$.

Now we recall from the previous derivation that $\overline{\mathcal{M}}$ is also skew-Hermitian. If the same assumptions described above hold for the nonlinearity in (28) (such is verifiably the case for the Boussinesq system) then we can again apply the same argument. Allowing the change of variables this time given by

$$
\overline{\boldsymbol{u}}(t, \tau, \alpha, \boldsymbol{x})=e^{\left(\tau-\frac{t}{\epsilon}\right) \overline{\mathcal{M}}} \hat{\boldsymbol{u}}(t, \alpha, \boldsymbol{x}),
$$

we see that $\overline{\boldsymbol{u}}$ satisfies (17) in the limit $\epsilon \rightarrow 0$, implying that the full solution can rigorously be shown to satisfy

$$
\boldsymbol{u}(t, x)=e^{-\frac{t}{\epsilon} \overline{\mathcal{M}}} e^{-\frac{t}{\delta} \mathcal{L}} \overline{\boldsymbol{u}}(t, \boldsymbol{x})+o(1),
$$

where $o(1)$ is referring to corrective terms of both $O(\epsilon)$ and $O(\delta)$. 
4.2 Renormalization group method

We now derive the reduced system (17) from the renormalization method (cf. [10] , [11], and [28]); our approach closely follows that in [44]. For simplicity, we neglect the dissipative operator $\mathcal{D}$ and consider the case $\delta=\epsilon^{1 / 2}$, so that

$$
\frac{\partial \mathbf{u}}{\partial t}+\frac{1}{\epsilon} \mathcal{L} \mathbf{u}+\frac{1}{\sqrt{\epsilon}} \mathcal{K} \mathbf{u}+\mathcal{B}(\mathbf{u}, \mathbf{u})=\mathbf{0} .
$$

Although the derivation is significantly longer than that obtained via multiple scales, no a priori assumption is needed on the form of time scale separation. We include a sketch of the derivation as the final result is the same as that achieved above via multiple scales.

We first put the system (31) into standard form with the change of variables, $\mathbf{u}(t)=e^{-\tau \mathcal{L}} \hat{\mathbf{u}}(\tau)$, where $\tau=t / \epsilon$. Then $\hat{\mathbf{u}}$ satisfies the equation

$$
\frac{\partial \hat{\mathbf{u}}}{\partial \tau}+\epsilon \mathcal{F}(\hat{\mathbf{u}}, \tau)+\sqrt{\epsilon \mathcal{M}}(\tau) \hat{\mathbf{u}}=\mathbf{0},
$$

where $\mathcal{M}(\tau)=e^{\tau \mathcal{L}} \mathcal{K} e^{-\tau \mathcal{L}}$ and

$$
\mathcal{F}(\mathbf{v}, \tau)=e^{\tau \mathcal{L}} \mathcal{B}\left(e^{-\tau \mathcal{L}} \mathbf{v}, e^{-\tau \mathcal{L}} \mathbf{v}\right)
$$

Following [44], we first consider the ordinary perturbation expansion (i.e., no multiple scales),

$$
\hat{\mathbf{u}}(\tau)=\hat{\mathbf{u}}_{0}(\tau)+\sqrt{\epsilon} \hat{\mathbf{u}}_{1}(\tau)+\epsilon \hat{\mathbf{u}}_{2}(\tau)+\ldots
$$

Then by substituting the above expansion into (32), we match powers of $\epsilon$ and solve for $\hat{\mathbf{u}}_{0}, \hat{\mathbf{u}}_{1}$, and $\hat{\mathbf{u}}_{2}$. In particular, define the linear operators

$$
\begin{gathered}
\overline{\mathcal{M}}=\lim _{T \rightarrow \infty} \frac{1}{T} \int_{0}^{T} \mathcal{M}(s) d s, \\
\mathcal{M}(s)=\overline{\mathcal{M}}+\partial_{s} \underline{\mathcal{M}(s)},
\end{gathered}
$$

and

$$
\mathcal{M}_{0}(\tau)=\frac{1}{2}\left({\underline{\mathcal{M}(\tau)^{2}}}^{2} \underline{\mathcal{M}(0)^{2}}\right)+\left(\underline{\mathcal{M}(0) \mathcal{M}(\tau)}-\underline{\mathcal{M}(0)^{2}}\right) .
$$

Also, define the nonlinear operators

$$
\overline{\mathcal{F}\left(\hat{\mathbf{u}}_{0}(\tau)\right)}=\lim _{T \rightarrow \infty} \frac{1}{T} \int_{0}^{T} \mathcal{F}\left(\hat{\mathbf{u}}_{0}(\tau), s\right) d s,
$$

and

$$
\mathcal{M}_{1}\left(\hat{\mathbf{u}}_{0}, \tau\right)=\mathcal{M}_{0}(\tau) \hat{\mathbf{u}}_{0}+\left(\underline{\mathcal{F}\left(\hat{\mathbf{u}}_{0}, \tau\right)}-\underline{\mathcal{F}\left(\hat{\mathbf{u}}_{0}, 0\right)}\right) \mathbf{v}_{0} .
$$

Then a straightforward (but tedious) calculation shows that, neglecting terms of $\operatorname{order} \mathcal{O}\left(\epsilon^{3 / 2}\right)$,

$$
\begin{array}{r}
\hat{\mathbf{u}}(\tau)=\hat{\mathbf{u}}_{0}-\sqrt{\epsilon}\left(\tau \overline{\mathcal{M}} \hat{\mathbf{u}}_{0}+(\underline{\mathcal{M}(\tau)}-\underline{\mathcal{M}(0)}) \hat{\mathbf{u}}_{0}\right) \\
-\epsilon\left(\frac{1}{2} \tau^{2} \overline{\mathcal{M}}^{2} \hat{\mathbf{u}}_{0}+\tau \overline{\mathcal{M}} \underline{\mathcal{M}(\tau)} \hat{\mathbf{u}}_{0}-\tau \overline{\mathcal{M}} \underline{\mathcal{M}(0)} \hat{\mathbf{u}}_{0}+\tau \overline{\mathcal{F}\left(\hat{\mathbf{u}}_{0}\right)}+\mathcal{M}_{1}\left(\hat{\mathbf{u}}_{0}, \tau\right) \hat{\mathbf{u}}_{0}\right) .
\end{array}
$$


Note that terms in the above asymptotic approximation grow in $\tau$. In particular, the $\epsilon^{1 / 2}$ terms are $\mathcal{O}(1)$ when $\tau \sim \epsilon^{-1 / 2}$ so that the approximation is no longer valid.

In order to obtain an asymptotic approximation that is valid on a longer time scale of $0 \leq \tau \leq \mathcal{O}\left(\epsilon^{-1 / 2}\right)$, we remove the secular terms (i.e. those that grow in $\tau$ ) via renormalization. To do so, we assume that there is a slowly varying function $\overline{\mathbf{v}}(\tau)$ such that $\overline{\mathbf{v}}(0)=\hat{\mathbf{u}}_{0}$. We then rewrite (32) in terms of $\overline{\mathbf{v}}$, and choose $\overline{\mathbf{v}}$ so that the secular terms are removed. More specifically, we use the Taylor expansion of $\overline{\mathbf{v}}(\tau)$ about $\tau=0$,

$$
\mathbf{v}_{0}=\overline{\mathbf{v}}(\tau-\tau)=\overline{\mathbf{v}}(\tau)-\tau \frac{d \overline{\mathbf{v}}}{d \tau}(\tau)+\frac{\tau^{2}}{2} \frac{d^{2} \overline{\mathbf{v}}}{d \tau^{2}}(\tau)+\ldots
$$

Then assuming that $\overline{\mathbf{v}}(\tau)$ satisfies

$$
\frac{d \overline{\mathbf{v}}}{d \tau}(\tau)+\sqrt{\epsilon} \mathcal{W}_{1}(\overline{\mathbf{v}})+\epsilon \mathcal{W}_{2}(\overline{\mathbf{v}})=\mathbf{0},
$$

we use (34) and (35) in equation (32) to obtain

$$
\begin{array}{r}
\left.\hat{\mathbf{u}}(\tau)=\overline{\mathbf{v}}+\tau \sqrt{\epsilon} \mathcal{W}_{1}(\overline{\mathbf{v}})-\epsilon \frac{\tau^{2}}{2} \frac{\delta \mathcal{W}_{1}}{\delta \mathbf{v}}(\overline{\mathbf{v}}) \mathcal{W}_{1}(\overline{\mathbf{v}})+\epsilon \tau \mathcal{W}_{2}((\hat{j}))^{6}\right) \\
-\sqrt{\epsilon}\left(\tau \overline{\mathcal{M}} \overline{\mathbf{v}}+\tau^{2} \sqrt{\epsilon \overline{\mathcal{M}}} \mathcal{W}_{1}(\overline{\mathbf{v}})+(\underline{\mathcal{M}(\tau)}-\underline{\mathcal{M}(0)}) \overline{\mathbf{v}}+(\underline{\mathcal{M}(\tau)}-\underline{\mathcal{M}(0)}) \tau \sqrt{\epsilon} \mathcal{W}_{1}(\overline{\mathbf{v}})\right) \\
-\epsilon\left(\frac{1}{2} \tau^{2} \overline{\mathcal{M}}^{2} \overline{\mathbf{v}}+\tau \overline{\mathcal{M}}(\underline{\mathcal{M}(\tau)}-\underline{\mathcal{M}(0)}) \overline{\mathbf{v}}+\tau \overline{\mathcal{F}(\overline{\mathbf{v}})}+\mathcal{M}_{1}(\overline{\mathbf{v}}, \tau) \overline{\mathbf{v}}\right)+\ldots
\end{array}
$$

In order to eliminate the secular term at order $\sqrt{\epsilon}$ we require that

$$
\mathcal{W}_{1}(\overline{\mathbf{v}})=\overline{\mathcal{M}} \overline{\mathbf{v}}
$$

It then follows that the secular terms in (36) at order $\epsilon$ are

$$
\begin{array}{r}
-\frac{\tau^{2}}{2} \overline{\mathcal{M}}^{2} \overline{\mathbf{v}}+\tau^{2} \overline{\mathcal{M}}^{2} \overline{\mathbf{v}}+(\underline{\mathcal{M}(\tau)}-\underline{\mathcal{M}(0)}) \tau \overline{\mathcal{M}} \overline{\mathbf{v}} \\
-\frac{1}{2} \tau^{2} \overline{\mathcal{M}}^{2} \overline{\mathbf{v}}-\tau \overline{\mathcal{M}}(\underline{\mathcal{M}(\tau)}-\underline{\mathcal{M}(0)}) \overline{\mathbf{v}}-\tau \overline{\mathcal{F}(\overline{\mathbf{v}})}+\tau \mathcal{W}_{2}(\overline{\mathbf{v}}) \\
=-\tau \overline{\mathcal{F}(\overline{\mathbf{v}})}+\tau \mathcal{W}_{2}(\overline{\mathbf{v}}) .
\end{array}
$$

We finally have that

$$
\mathcal{W}_{2}(\overline{\mathbf{v}})=\overline{\mathcal{F}(\overline{\mathbf{v}})}
$$

In total,

$$
\frac{d \overline{\mathbf{v}}}{d \tau}(\tau)+\sqrt{\epsilon \overline{\mathcal{M}}} \overline{\mathbf{v}}+\epsilon \overline{\mathcal{F}(\overline{\mathbf{v}})}=\mathbf{0}
$$

The same renormalization procedure can again be applied to equation (37), in order to obtain a reduced equation that is independent of $\epsilon$. The result will be the same averaged equation (17) obtained via multiple scales. In particular, let $\alpha=\sqrt{\epsilon} \tau$ and $\overline{\mathbf{w}}(\tau)=e^{-\alpha \overline{\mathcal{M}}} \overline{\mathbf{v}}(\alpha)$, and rewrite (37) as

$$
\frac{d \overline{\mathbf{w}}}{d \alpha}=\epsilon^{1 / 2} \mathcal{G}(\overline{\mathbf{w}}, \alpha)
$$


where

$$
\mathcal{G}(\overline{\mathbf{w}}, \alpha)=e^{\alpha \overline{\mathcal{M}}} \overline{\mathcal{F}\left(e^{-\alpha \overline{\mathcal{M}}} \overline{\mathbf{w}}\right)}
$$

Going through the same procedure as above, we see that

$$
\overline{\mathbf{w}}(\alpha)=\overline{\mathbf{z}}(\alpha)+\mathcal{O}(\epsilon),
$$

where $\overline{\mathbf{z}}$ satisfies

$$
\begin{aligned}
\frac{d \overline{\mathbf{z}}}{d \alpha} & =\epsilon^{1 / 2} \lim _{T_{2} \rightarrow \infty} \frac{1}{T_{2}} \int_{0}^{T_{2}} \mathcal{G}(\overline{\mathbf{z}}(\alpha), r) d r \\
& =\epsilon^{1 / 2} \lim _{T_{2} \rightarrow \infty} \frac{1}{T_{2}} \int_{0}^{T_{1}}\left(\lim _{T_{1} \rightarrow \infty} \frac{1}{T_{1}} \int_{0}^{T_{1}} e^{r \overline{\mathcal{M}}} e^{s \mathcal{L}} \mathcal{B}\left(e^{-s \mathcal{L}} e^{-r \overline{\mathcal{M}}} \overline{\mathbf{z}}(\alpha), e^{-s \mathcal{L}} e^{-r \overline{\mathcal{M}}} \overline{\mathbf{z}}(\alpha)\right) d s d r\right) .
\end{aligned}
$$

In the last equality, we used (33). Finally, defining $\overline{\mathbf{u}}(t)=\overline{\mathbf{z}}(s)$, and using that $t=\epsilon \tau=\sqrt{\epsilon} s$ and $e^{-s \mathcal{L}} e^{-r \overline{\mathcal{M}}}=e^{-r \overline{\mathcal{M}}} e^{-s \mathcal{L}}$, (from the commutativity of $\mathcal{L}$ and $\overline{\mathcal{M}}$ as shown in the Appendix) we find that

$$
\begin{array}{r}
\mathbf{u}(t)=e^{-\tau \mathcal{L}} \overline{\mathbf{v}}(\tau)+\mathcal{O}(\epsilon) \\
=e^{-\tau \mathcal{L}} e^{-\alpha \overline{\mathcal{M}}} \overline{\mathbf{w}}(\alpha)+\mathcal{O}(\epsilon) \\
=e^{-(t / \epsilon) \mathcal{L}} e^{-(t / \sqrt{\epsilon}) \overline{\mathcal{M}}} \overline{\mathbf{u}}(t)+\mathcal{O}(\epsilon),
\end{array}
$$

where $\overline{\mathbf{u}}(t)$ satisfies the system (17).

\subsection{Cautionary tales of asymptotic generalizations}

While the method of cancellation of oscillations provides rigorous results for the asymptotics, in this case it is less clear when that rigor can be applied. Explicitly, as demonstrated above the limiting system for the slow dynamics in (17) is valid if the two different limits can be taken independently. It is clear then that the multiple scale calculation carried out in Sections 2 and 3 is equivalent to deriving the asymptotic system for the fastest time scale first, and then performing a similar asymptotic derivation on the next 'fastest' time scale. What is remarkable is that as noted in Section 2 (and supported by the renormalization calculation above, although such is demonstrated only for the quadratic relation), the multiple scales calculation indicates that such a reduced system describes the evolution of the flow on the $O(1)$ 'slow manifold' even when the small parameters are related via a power law. However, this result is not supported directly by the rigorous theory of the cancellation of oscillations which demands that the two limits remain distinct. The cancellation of oscillations as outlined here relies on the smallest parameter $\delta$ completely vanishing before considering the limit $\epsilon \rightarrow 0$, while the derivation for multiple scales assumes that $\delta, \epsilon \rightarrow 0$ simultaneously.

This sequential application of the asymptotic limits must be considered with caution as it is not generically the case that $\overline{\mathcal{M}}$ is equivalent to the projection of $\mathcal{K}$ onto the $\mathcal{L}$ slow manifold. On the contrary, if we consider the notation developed in [14], and using the skew-Hermitian property of $\mathcal{L}$, then we can write the $O(1)$ solution as

$$
\boldsymbol{u}_{0}=\hat{\boldsymbol{u}}_{0}^{S}+e^{-\alpha \mathcal{L}} \hat{\boldsymbol{u}}_{0}^{F}
$$


where $\hat{\boldsymbol{u}}_{0}^{S}$ lies in the null space of $\mathcal{L}$. The projection of $\mathcal{K}$ onto the $\mathcal{L}$ slow manifold would be achieved by restricting $\boldsymbol{u}_{0}$ to $\hat{\boldsymbol{u}}_{0}^{S}$ and neglecting the influence of the $\alpha$-fast part of the solution. In contrast the linear operator $\overline{\mathcal{M}}$ as defined above allows for the interaction of the $\mathcal{L}$-fast waves with the $\mathcal{K}$-slow parts of the flow. Symbolically if we let $P_{\mathcal{L}}$ be the projection operator onto the null space of $\mathcal{L}$ then we may state that $\overline{\mathcal{M}} \boldsymbol{u}_{0} \neq P_{\mathcal{L}} \mathcal{K} P_{\mathcal{L}} \boldsymbol{u}_{0}$ unless $P \boldsymbol{u}_{0}=\boldsymbol{u}_{0}$. In essence then, there is a distinctive difference between projecting the solution onto the slow manifold defined by a single fast variable, and one defined by multiple ones.

There is no clear extension of the theory developed by [34] to the case of two fast, functionally related time scales. For example, if there is a quadratic relation between $\delta$ and $\epsilon$ and the naive change of variables

$$
\overline{\boldsymbol{u}}(t, \tau, \alpha, \boldsymbol{x})=e^{\left(\tau-\frac{t}{\epsilon}\right)} \overline{\mathcal{M}}_{e}^{\left(\alpha-\frac{t}{\epsilon^{2}}\right) \mathcal{L}} \boldsymbol{u}(t, \boldsymbol{x})
$$

is assumed, one cannot even establish that $\frac{\partial \overline{\boldsymbol{u}}}{\partial t}$ is bounded, let alone prove a double averaging lemma akin to Lemma 2.2 in [34]. Hence, although the multiple scales calculation indicates that the reduced system (17) is valid for all small parameters related via a power relation, such an assertion should be taken with caution until a more rigorous statement can be made.

The multiple scales calculation by itself indicates that the final result is not as robust as would be desired. In the first place, as mentioned in Section 2 the error to the $O(1)$ system described by (17) and (11) is on the order of the larger of the two small parameters, indicating that the accuracy of the asymptotics is dictated by the slowest of the two fast time-scales. In addition, as noted particularly in Section 3 and observed in the renormalization calculation above, even though the small parameters are assumed to be functionally related via a power law, the potentially secular terms are not removed from the calculation without assuming complete separation of scales, i.e. $\epsilon \rightarrow 0$ or $\delta \rightarrow 0$. In summary the slow dynamics governed by (17) may not be as widely applicable as the single limit asymptotics. Even so, the primary takeaway from the present derivation is that the dual limit $\epsilon \rightarrow 0, \frac{\epsilon}{\delta}=O(1)$ is not the only possibility when two fast time scales are present.

\section{The rotating, stratified Boussinesq system}

\subsection{Overview of the full system}

As a specific example of the derivation provided above we will consider the nondimensional rotating, stratified Boussinesq equations (see $[14,43]$ ):

$$
\begin{aligned}
\frac{\partial \boldsymbol{v}}{\partial t}+\boldsymbol{v} \cdot \nabla \boldsymbol{v}+\frac{1}{\operatorname{Ro}} \hat{z} \times \boldsymbol{v} & +\nabla \Delta^{-1}\left(\frac{1}{\operatorname{Ro}} \hat{z} \cdot \boldsymbol{\omega}-\frac{1}{\operatorname{Fr}} \frac{\partial \rho}{\partial z}-\nabla \cdot(\boldsymbol{v} \cdot \nabla \boldsymbol{v})\right)+\frac{1}{\mathrm{Fr}} \hat{z} \rho(42) \\
& =\frac{1}{\operatorname{Re}} \Delta \boldsymbol{v} \\
\nabla \cdot \boldsymbol{v} & =0 \\
\frac{\partial \rho}{\partial t}+\boldsymbol{v} \cdot \nabla \rho & =\frac{1}{\operatorname{RePr}} \Delta \rho-\frac{1}{\mathrm{Fr}} w
\end{aligned}
$$


In order to achieve the format of (1) the pressure was eliminated via the incompressibility constraint. Thus, allowing $\boldsymbol{u}=(\boldsymbol{v}, \rho)^{T}, \epsilon=\operatorname{Ro}, \delta=$ Fr and

$$
\begin{array}{r}
\mathcal{L} \boldsymbol{u}=\left(\begin{array}{c}
\hat{z} \rho-\nabla \Delta^{-1}\left(\frac{\partial \rho}{\partial z}\right) \\
-w
\end{array}\right) \quad \mathcal{K} \boldsymbol{u}=\left(\begin{array}{c}
\hat{z} \times \boldsymbol{v}+\nabla \Delta^{-1} \omega \\
0
\end{array}\right) \\
\mathcal{B}(\boldsymbol{u}, \boldsymbol{u})=\left(\begin{array}{c}
\boldsymbol{v} \cdot \nabla \boldsymbol{v}-\nabla \Delta^{-1}(\nabla \cdot(\boldsymbol{v} \cdot \nabla \boldsymbol{v})) \\
\boldsymbol{v} \cdot \nabla \rho
\end{array}\right) \quad \mathcal{D} \boldsymbol{u}=\left(\begin{array}{c}
\frac{1}{\operatorname{Re}} \Delta \boldsymbol{v} \\
\frac{1}{\operatorname{RePr}} \Delta \rho
\end{array}\right),
\end{array}
$$

we see that the derivation provided in the earlier Sections can be applied directly to this system. The velocity field $\boldsymbol{v}=(u, v, w)^{T}$ and density variable $\rho$ are the prognostic variables, with $\boldsymbol{\omega}=\nabla \times \boldsymbol{v}$ being the vorticity, and $\hat{z}$ referring to the vertical unit vector. The non-dimensional numbers of the system are the Rossby number Ro measuring the strength of the effective rotation, the Froude number Fr measuring the strength of the stratification, the Reynold's number Re measuring the relative strength of the flow to the viscous dissipation, and the Prandtl number Pr that measures the relative strength of the viscous dissipation to the thermal diffusivity.

Before considering the relative effects of taking $\mathrm{Fr} \rightarrow 0$ (strong stratification) and Ro $\rightarrow 0$ (rapid rotation) we note that the separate limits of these two cases as well as the case of both parameters vanishing with $\frac{\mathrm{Ro}}{\mathrm{Fr}}=O(1)$ are considered in detail in $[14,43]$. Following their lead, we will consider a triply periodic geometry on the unit cube $[0,1]^{3}$. For completeness and reference for the following derivation, we first recall the Fourier representation (of value in this triply periodic space) of each of the linear operators, and their eigen-decomposition. Details for each case are given in $[14,43]$. In addition we include information relative to the projection operator onto the null space of each of these linear operators. This is used to determine an explicit set of equations for the slow limiting dynamics, and represents the operator that takes the full solution to the $O(1)$ effective slow dynamics (ignoring the effect of the fast waves).

\subsection{Decomposition of the rotation operator}

In the limit of rapid rotation as discussed in [43], it is necessary to explicitly evaluate the null space of the 'fast' operator $\mathcal{K}$. This leads to the projection operator $P_{\mathcal{K}}$ onto this space, given as eqn. (3.17) in [43] and repeated here for completeness:

$$
P_{\mathcal{K}} \boldsymbol{u}=\left(\begin{array}{c}
\left\langle\boldsymbol{v}_{H}\right\rangle_{z}-\nabla_{H} \Delta_{H}^{-1}\left(\nabla_{H} \cdot\left\langle\boldsymbol{v}_{H}\right\rangle_{z}\right) \\
\langle w\rangle_{z} \\
\rho
\end{array}\right)
$$

where $\langle\cdot\rangle_{z}$ denotes the vertical average over the domain, and the ${ }_{H}$ indicates the horizontal component only, i.e. $\Delta_{H}=\partial_{x x}+\partial_{y y}$ and $\boldsymbol{v}_{H}=(u, v)^{T}$. This indicates that in the limit of rapid rotation (and weak stratification) that the slow dynamics are governed by 2D Navier-Stokes coupled to a two-scalar interaction between density and vertically averaged vertical velocity (see [43]). 
In terms of the Fourier modes $\boldsymbol{u}_{k}(\boldsymbol{x})=e^{\imath \boldsymbol{k} \cdot \boldsymbol{x}} \boldsymbol{r}_{k}$ the rotating linear operator can be expressed as

$\mathcal{K}(\boldsymbol{k})=\frac{1}{|\boldsymbol{k}|^{2}}\left(\begin{array}{cccc}-k l & -\left(l^{2}+m^{2}\right) & 0 & 0 \\ k^{2}+m^{2} & k l & 0 & 0 \\ -l m & k m & 0 & 0 \\ 0 & 0 & 0 & 0\end{array}\right), \quad \mathcal{K}(0)=\left(\begin{array}{cccc}0 & -1 & 0 & 0 \\ 1 & 0 & 0 & 0 \\ 0 & 0 & 0 & 0 \\ 0 & 0 & 0 & 0\end{array}\right)$

where $\boldsymbol{k}=(k, l, m)^{T}$ is the three-dimensional wave-number and on a wave-number by wave-number basis the action of $\mathcal{K}$ on $\boldsymbol{u}_{\boldsymbol{k}}(\boldsymbol{x})$ can be calculated as

$$
\mathcal{K} \boldsymbol{u}_{k}(\boldsymbol{x})=e^{\imath \boldsymbol{k} \cdot \boldsymbol{x}} \mathcal{K}(\boldsymbol{k}) \boldsymbol{r}_{k}
$$

The eigen-decomposition of $\mathcal{K}$ provides insight into the effective nature of the slow and fast components of the flow. There are four imaginary eigenvalues given by $\lambda_{k}^{\mathcal{K}}=\imath \omega_{k}^{\mathcal{K}, \beta}$ where $\omega_{k}^{\mathcal{K}, \pm 1}= \pm \frac{m}{|\boldsymbol{k}|}$ corresponds to the fast inertial modes, and $\omega_{k}^{\mathcal{K}, 0}=0$ corresponds to the slow modes of the system. For $\left|\boldsymbol{k}_{H}\right| \neq 0$ this leads to the following eigenvectors

$$
\boldsymbol{r}_{k}^{\mathcal{K}, \pm 1}=\frac{1}{\sqrt{2}\left|\boldsymbol{k}_{H}\right||\boldsymbol{k}|}\left(\begin{array}{c}
\mp l|\boldsymbol{k}| \pm \imath k m \\
\pm k|\boldsymbol{k}| \pm \imath l m \\
-\imath\left|\boldsymbol{k}_{H}\right|^{2} \\
0
\end{array}\right), \quad \boldsymbol{r}_{k}^{\mathcal{K}, 0}=\left(\begin{array}{l}
0 \\
0 \\
0 \\
1
\end{array}\right)
$$

where the fourth eigenvector violates the incompressibility condition $\left(\boldsymbol{v}_{k} \cdot \boldsymbol{k}=0\right.$ in Fourier space). This eigenvector can be neglected, as incompressibility reduces the dimension of the system by one (the presentation via a $4 \times 4$ system is for pedagogical purposes). When $\left|\boldsymbol{k}_{H}\right|=0$ we have

$$
\boldsymbol{r}_{k}^{\mathcal{K}, \pm 1}=\frac{1}{\sqrt{2}}\left(\begin{array}{c}
1 \\
\mp \imath \\
0 \\
0
\end{array}\right), \quad \boldsymbol{r}_{k}^{\mathcal{K}, 0}=\left(\begin{array}{c}
0 \\
0 \\
0 \\
1
\end{array}\right), \quad \tilde{\boldsymbol{r}}_{k}^{\mathcal{K}, 0}=\left(\begin{array}{c}
0 \\
0 \\
1 \\
0
\end{array}\right)
$$

where $\tilde{\boldsymbol{r}}_{k}^{\mathcal{K}, 0}$ is valid only when $|\boldsymbol{k}|=0$, and the eigenvalues for the fast modes are now dictated by $\omega_{k}^{\mathcal{K}, \pm 1}= \pm 1$.

\subsection{Decomposition of the stratification operator}

As outlined in [14] the projection onto the null space of $\mathcal{L}$ is given by

$$
P_{\mathcal{L}} \boldsymbol{u}=\left(\begin{array}{c}
\boldsymbol{v}_{H}-\nabla_{H} \Delta_{H}^{-1}\left(\nabla_{H} \cdot \boldsymbol{v}_{H}\right) \\
0 \\
\langle\rho\rangle_{H}
\end{array}\right)
$$

where $\langle\cdot\rangle_{H}$ here corresponds to the horizontal average. As indicated in [14] and explored through numerical experiments (see $[24,27,36,37,33]$ ) this implies that in the limit of strong stratification and weak rotation the flow qualitatively develops into pancake-like structures with little to no influence from the vertical velocity, but significant vertical shear in the horizontal components of momentum. 
In Fourier space, the linear stratification operator is

$$
\mathcal{L}(\boldsymbol{k})=\left(\begin{array}{cccc}
0 & 0 & 0 & -\frac{k m}{|\boldsymbol{k}|^{2}} \\
0 & 0 & 0 & -\frac{l m}{|\boldsymbol{k}|^{2}} \\
0 & 0 & 0 & 1-\frac{m^{2}}{|\boldsymbol{k}|^{2}} \\
0 & 0 & -1 & 0
\end{array}\right), \quad \mathcal{L}(0)=\left(\begin{array}{cccc}
0 & 0 & 0 & 0 \\
0 & 0 & 0 & 0 \\
0 & 0 & 0 & 1 \\
0 & 0 & -1 & 0
\end{array}\right)
$$

with purely imaginary eigenvalues for the fast waves with frequency $\omega_{k}^{\mathcal{L}, \pm 1}=$ $\mp \frac{\left|\boldsymbol{k}_{H}\right|}{|\boldsymbol{k}|}$, and slow $\omega^{\mathcal{L}, 0}=0$ which for $\left|\boldsymbol{k}_{H}\right| \neq 0$ has corresponding eigenvectors

$$
\boldsymbol{r}_{k}^{\mathcal{L}, \pm 1}=\frac{1}{\sqrt{2}}\left(\begin{array}{c}
\mp \imath \frac{k m}{\left|\boldsymbol{k}_{H}\right||\boldsymbol{k}|} \\
\mp \imath \frac{l m}{\left|\boldsymbol{k}_{H}\right||\boldsymbol{k}|} \\
\pm \imath \frac{|\boldsymbol{k}|}{|\boldsymbol{k}|} \\
1
\end{array}\right), \quad \boldsymbol{r}_{k}^{\mathcal{L}, 0}=\frac{\imath}{\left|\boldsymbol{k}_{H}\right|}\left(\begin{array}{c}
-l \\
k \\
0 \\
0
\end{array}\right)
$$

When $\left|\boldsymbol{k}_{H}\right|=0$ all of the eigenvalues are zero, and for $m \neq 0$ the corresponding eigenvectors are

$$
\boldsymbol{r}_{k}^{\mathcal{L}, \pm 1}=\frac{1}{\sqrt{2}}\left(\begin{array}{c} 
\pm \imath \\
1 \\
0 \\
0
\end{array}\right), \quad \boldsymbol{r}_{k}^{\mathcal{L}, 0}=\left(\begin{array}{l}
0 \\
0 \\
0 \\
1
\end{array}\right)
$$

and when $|\boldsymbol{k}|=0, \quad \omega_{0}^{\mathcal{L}, \pm 1}=\mp 1$ and

$$
\boldsymbol{r}_{0}^{\mathcal{L}, \pm 1}=\frac{1}{\sqrt{2}}\left(\begin{array}{c}
0 \\
0 \\
\mp \imath \\
1
\end{array}\right), \quad \boldsymbol{r}_{0}^{\mathcal{L}, 0}=\left(\begin{array}{l}
1 \\
0 \\
0 \\
0
\end{array}\right), \quad \tilde{\boldsymbol{r}}_{0}^{\mathcal{L}, 0}=\left(\begin{array}{l}
0 \\
1 \\
0 \\
0
\end{array}\right)
$$

5.4 Rapid rotation dominating strong stratification in the Boussinesq system

We will first consider the situation when the rotation is asymptotically stronger than the asymptotically strong stratification. The opposite situation is considered in Section 5.5. First, we compute the rotationally averaged stratification operator $\overline{\mathcal{M}}$ for this case as well as the resultant slow system by projecting onto the null space of both $\mathcal{K}$ and $\overline{\mathcal{M}}$. We then demonstrate that in this limit, just as in the rapidly rotating weakly stratified (see [43]) limit, there is no fast-fast-slow wave interaction.

5.4.1 The stratification operator averaged on a rapidly rotating time scale and the resultant slow equations

We first explicitly compute $\overline{\mathcal{M}}$ (here the roles of $\mathcal{K}$ and $\mathcal{L}$ as defined in (45) are reversed). To illustrate how this is done, we expand the dependent variables in terms of the eigenfunctions of the fastest operator $\mathcal{K}$

$$
\boldsymbol{u}=\sum_{\gamma, k} \sigma_{k}^{\mathcal{K}, \gamma}(t) e^{\imath \boldsymbol{k} \cdot \boldsymbol{x}} \boldsymbol{r}_{k}^{\mathcal{K}, \gamma}
$$


where the $\boldsymbol{r}_{k}^{\mathcal{K}, \gamma}$ are given in Section 5.2. Following [13,14,43] we note that

$$
e^{s \mathcal{K}}\left(e^{\imath \boldsymbol{k} \cdot \boldsymbol{x}} \boldsymbol{r}_{k}^{\mathcal{K}, \gamma}\right)=e^{\imath s \omega_{k}^{\mathcal{K}, \gamma}} e^{\imath \boldsymbol{k} \cdot \boldsymbol{x}} \boldsymbol{r}_{k}^{\mathcal{K}, \gamma}
$$

Noting that any vector $\boldsymbol{x}$ can be written as

$$
\boldsymbol{x}=\sum_{\gamma, k}\left\langle\boldsymbol{x}, \boldsymbol{r}_{k}^{\mathcal{K}, \gamma}\right\rangle \boldsymbol{r}_{k}^{\mathcal{K}, \gamma}
$$

where $\langle\cdot, \cdot\rangle$ is the appropriate inner product, we recognize that $\overline{\mathcal{M}} \boldsymbol{x}$ can be determined by computing $\overline{\mathcal{M}} \boldsymbol{r}_{k}^{\mathcal{K}, \gamma}$ for each eigenvector $\boldsymbol{r}_{k}^{\mathcal{K}, \gamma}$ of $\mathcal{K}$.

In order to proceed we compute the action of $\overline{\mathcal{M}}$ on each of the eigenvectors of $\mathcal{K}$ on a case by case basis.

1. $\left|\boldsymbol{k}_{H}\right| \neq 0$. In this case the eigenvectors are given by (50) and we have the following

$$
\begin{aligned}
& \mathcal{L} \boldsymbol{r}_{k}^{\mathcal{K}, \pm 1}=\frac{\imath\left|\boldsymbol{k}_{H}\right|}{\sqrt{2}|\boldsymbol{k}|}\left(\begin{array}{c}
0 \\
0 \\
0 \\
1
\end{array}\right)=\frac{\imath\left|\boldsymbol{k}_{H}\right|}{\sqrt{2}|\boldsymbol{k}|} \boldsymbol{r}_{k}^{\mathcal{K}, 0} \\
& \Rightarrow e^{s \mathcal{K}} \mathcal{L} e^{-s \mathcal{K}}\left(e^{\imath \boldsymbol{k} \cdot \boldsymbol{x}} \boldsymbol{r}_{k}^{\mathcal{K}, \pm 1}\right)=\frac{\imath\left|\boldsymbol{k}_{H}\right|}{\sqrt{2}|\boldsymbol{k}|} e^{-\imath \frac{m}{|\boldsymbol{k}|} s} e^{\imath \boldsymbol{k} \cdot \boldsymbol{x}} \boldsymbol{r}_{k}^{\mathcal{K}, 0} \\
& \Rightarrow \overline{\mathcal{M}}\left(e^{\imath \boldsymbol{k} \cdot \boldsymbol{x}} \boldsymbol{r}^{\mathcal{K}, \pm 1}\right)=0 \text {. } \\
& \mathcal{L} \boldsymbol{r}_{k}^{\mathcal{K}, 0}=\frac{1}{|\boldsymbol{k}|^{2}}\left(\begin{array}{c}
k m \\
l m \\
\left|\boldsymbol{k}_{H}\right|^{2} \\
0
\end{array}\right) \\
& =\frac{\imath\left|\boldsymbol{k}_{H}\right|}{\sqrt{2}|\boldsymbol{k}|}\left(\frac{m^{2}-\left|\boldsymbol{k}_{H}\right|^{2}}{|\boldsymbol{k}|^{2}} \boldsymbol{r}_{k}^{\mathcal{K}, 1}-\boldsymbol{r}_{k}^{\mathcal{K},-1}\right) \\
& \Rightarrow e^{s \mathcal{K}} \mathcal{L} e^{-s \mathcal{K}}\left(e^{\imath \boldsymbol{k} \cdot \boldsymbol{x}} \boldsymbol{r}_{k}^{\mathcal{K}, 0}\right)=\frac{\imath\left|\boldsymbol{k}_{H}\right|}{\sqrt{2}|\boldsymbol{k}|} e^{\imath \boldsymbol{k} \cdot \boldsymbol{x}}\left(\frac{m^{2}-\left|\boldsymbol{k}_{H}\right|^{2}}{|\boldsymbol{k}|^{2}} e^{\imath \frac{m}{|\boldsymbol{k}|} s} \boldsymbol{r}_{k}^{\mathcal{K}, 1}-e^{-\imath \frac{m}{|\boldsymbol{k}|} s} \boldsymbol{r}_{k}^{\mathcal{K},-1}\right) \\
& \Rightarrow \overline{\mathcal{M}}\left(e^{\imath \boldsymbol{k} \cdot \boldsymbol{x}} \boldsymbol{r}_{\mathcal{K}, k}^{0}\right)=0,
\end{aligned}
$$

indicating that $\overline{\mathcal{M}}$ will only affect the horizontally averaged components of the flow.

2. $\left|\boldsymbol{k}_{H}\right|=0$, but $m \neq 0$. Here we refer to (51) for the definition of the eigenvectors, and note that

$$
\begin{aligned}
\mathcal{L} \boldsymbol{r}_{k}^{\mathcal{K}, \pm 1}=0 & \Rightarrow \overline{\mathcal{M}} \boldsymbol{r}_{k}^{\mathcal{K}, \pm}=0 \\
\mathcal{L} \boldsymbol{r}_{k}^{\mathcal{K}, 0}=0 & \Rightarrow \overline{\mathcal{M}} \boldsymbol{r}_{k}^{\mathcal{K}, 0}=0
\end{aligned}
$$

so that $\overline{\mathcal{M}}$ only acts on the complete spatial average of the dependent variables. 3. $|\boldsymbol{k}|=0$. Again, we refer to (51) to see that

$$
\begin{array}{r}
\mathcal{L} \boldsymbol{r}_{0}^{\mathcal{K}, \pm 1}=0 \Rightarrow \overline{\mathcal{M}} \boldsymbol{r}_{0}^{\mathcal{K}, \pm 1}=0 \\
\mathcal{L} \boldsymbol{r}_{0}^{\mathcal{K}, 0}=\tilde{\boldsymbol{r}}_{0}^{\mathcal{K}, 0} \Rightarrow \overline{\mathcal{M}}\left(e^{\imath \boldsymbol{k} \cdot \boldsymbol{x}} \boldsymbol{r}_{k}^{\mathcal{K}, 0}\right)=\tilde{\boldsymbol{r}}_{0}^{\mathcal{K}, 0}, \\
\mathcal{L} \tilde{r}_{0}^{\mathcal{K}, 0}=-\boldsymbol{r}_{0}^{\mathcal{K}, 0} \Rightarrow \overline{\mathcal{M}}\left(e^{\left.\imath \boldsymbol{k} \cdot \boldsymbol{x}_{\tilde{\boldsymbol{r}}_{k}^{\mathcal{K}} .0}\right)=-\boldsymbol{r}_{0}^{\mathcal{K}, 0}} .\right.
\end{array}
$$


Putting this all together we see that we can write the linear operator $\overline{\mathcal{M}}$ in the case that rotation is the faster operator, as

$$
\overline{\mathcal{M}} \boldsymbol{u}=e^{\imath \boldsymbol{k} \cdot \boldsymbol{x}} \sum_{\gamma, k}\left\langle\boldsymbol{u}, \boldsymbol{r}_{k}^{\mathcal{K}, \gamma}\right\rangle \overline{\mathcal{M}} \boldsymbol{r}_{k}^{\mathcal{K}, \gamma}=\left\langle\boldsymbol{u}, \boldsymbol{r}_{0}^{\mathcal{K}, 0}\right\rangle \tilde{\boldsymbol{r}}_{0}^{\mathcal{K}, 0}-\left\langle\boldsymbol{u}, \tilde{\boldsymbol{r}}_{0}^{\mathcal{K}, 0}\right\rangle \boldsymbol{r}_{0}^{\mathcal{K}, 0}
$$

which, in real space becomes

$$
\overline{\mathcal{M}} \boldsymbol{u}=\left(\begin{array}{c}
0 \\
\langle\rho\rangle \\
-\langle w\rangle
\end{array}\right)
$$

where $\langle\cdot\rangle$ refers to the global spatial average. We can also readily compute the projection operator onto the null space of this operator as

$$
P_{\overline{\mathcal{M}}} \boldsymbol{u}=\left(\begin{array}{c}
\boldsymbol{v}_{H} \\
w-\langle w\rangle \\
\rho-\langle\rho\rangle
\end{array}\right) .
$$

We observe that this indicates that $\overline{\mathcal{M}}$ reduces to $\mathcal{L}$ in the mean, i.e. $\overline{\mathcal{M}}$ acts as the null operator for all $|\boldsymbol{k}| \neq 0$, and as $\mathcal{L}$ when $|\boldsymbol{k}|=0$.

The separation of the two fast time scales indicates that the $O(1)$ slow system can be found by projecting the flow onto the null space of $\mathcal{K}$ and then onto the null space of $\overline{\mathcal{M}}$. The evolution equation for this component of the flow is obtained by projecting the nonlinear and dissipative operators sequentially onto the null spaces of $\mathcal{K}$ and then $\overline{\mathcal{M}}$. The dissipation for the Boussinesq equations is trivial as $\mathcal{D}$ is a diagonal, linear operator, and hence is not affected by either $\mathcal{K}$ or $\overline{\mathcal{M}}$. We note that these projections are not equivalent to projecting the full evolution equation onto the null space of $\mathcal{K}$ and then applying another separation of scales argument. For rotation dominating stratification, the evolution of the flow is given by:

$$
\begin{aligned}
\frac{\partial \boldsymbol{v}_{H}^{S}}{\partial t}+\boldsymbol{v}_{H}^{S} \cdot \nabla \boldsymbol{v}_{H}^{S}-\nabla_{H} \Delta_{H}^{-1}\left(\nabla \cdot\left(\boldsymbol{v}_{H}^{S} \cdot \nabla \boldsymbol{v}_{H}^{S}\right)\right) & =\frac{1}{\operatorname{Re}} \Delta_{H} \boldsymbol{v}_{H}^{S}, \\
\nabla_{H} \cdot \boldsymbol{v}_{H}^{S} & =0, \\
\frac{\partial w}{\partial t}+\boldsymbol{v}_{H}^{S} \cdot \nabla_{H} w^{S} & =\frac{1}{\operatorname{Re}} \Delta_{H} w^{S}, \\
\frac{\partial \rho^{S}}{\partial t}+\boldsymbol{v}^{S} \cdot \nabla \rho^{S} & =\frac{1}{\operatorname{Re} \operatorname{Pr}} \Delta \rho^{S},
\end{aligned}
$$

where $\boldsymbol{v}^{S}=\left(\boldsymbol{v}_{H}^{S}, w^{S}\right)^{T}$ and

$$
\begin{aligned}
\boldsymbol{v}_{H}^{S} & =\langle\boldsymbol{v}\rangle_{z}, \\
w^{S} & =\langle w\rangle_{z}-\langle w\rangle, \\
\rho^{S} & =\rho-\langle\rho\rangle .
\end{aligned}
$$

Thus, on the slow manifold the system obeys the two-dimensional Navier-Stokes equations with two semi-active scalars that depend on the full three-dimensional fluid velocity. This is identical to the case of rapid rotation with weak stratification (see [43]) except that the buoyancy and vertical velocity variables have mean zero, and are nearly decoupled. 
5.4.2 Interactions between the fast and slow components of the flow

Following the notation introduced in $[14,43]$ we consider the decomposition of the flow as given by (57) where again the eigenvector expansion is taken from the fastest operator $\mathcal{K}$. Inserting this into the slow $O(1)$ equation (17) (again recalling that in this case $\mathcal{L}$ and $\mathcal{K}$ have reversed roles) we can after averaging over the 2 fast times, determine the evolution of each of the Fourier modes.

This done by first considering the effect of the nonlinear term $\mathcal{B}$ on the eigenvector decomposition of the flow:

$$
\begin{gathered}
\mathcal{B}\left(e^{-\beta \overline{\mathcal{M}}} e^{-s \mathcal{K}} \boldsymbol{u}, e^{-\beta \overline{\mathcal{M}}} e^{-s \mathcal{K}} \boldsymbol{u}\right) \\
=\mathcal{B}\left(e^{-\beta \overline{\mathcal{M}}}\left[\sum_{k \neq 0, \gamma} \sigma_{k}^{\mathcal{K}, \gamma}(t) e^{-\imath s \omega_{k}^{\mathcal{K}, \gamma}} \boldsymbol{r}_{k}^{\mathcal{K}, \gamma}+\sum_{\gamma} \sigma_{0}^{\mathcal{K}, \gamma}(t) e^{-\imath s \omega_{0}^{\mathcal{K}, \gamma}} \boldsymbol{r}_{0}^{\mathcal{K}, \gamma}\right],\right. \\
\left.e^{-\beta \overline{\mathcal{M}}}\left[\sum_{k \neq 0, \gamma} \sigma_{k}^{\mathcal{K}, \gamma}(t) e^{-\imath s \omega_{k}^{\mathcal{K}, \gamma}} \boldsymbol{r}_{k}^{\mathcal{K}, \gamma}+\sum_{\gamma} \sigma_{0}^{\mathcal{K}, \gamma}(t) e^{-\imath s \omega_{0}^{\mathcal{K}, \gamma}} \boldsymbol{r}_{0}^{\mathcal{K}, \gamma}\right]\right) .
\end{gathered}
$$

In order to proceed we first note that as shown above, for all $k \neq 0$ then $\overline{\mathcal{M}}=0$ and hence $e^{-\beta \overline{\mathcal{M}}}=I$. In addition, as shown above, when $k=0$ then $\overline{\mathcal{M}}=\mathcal{L}$ so that we may compute the following:

$$
\begin{aligned}
\boldsymbol{r}_{0}^{\mathcal{K}, \pm 1} & =\frac{1}{\sqrt{2}}\left[\boldsymbol{r}_{0}^{\mathcal{L}, 0} \mp \imath \tilde{\boldsymbol{r}}_{0}^{\mathcal{L}, 0}\right] \\
\Rightarrow e^{-\beta \overline{\mathcal{M}}} \boldsymbol{r}_{0}^{\mathcal{K}, \pm 1} & =\frac{1}{\sqrt{2}}\left[\boldsymbol{r}_{0}^{\mathcal{L}, 0} \mp \imath \tilde{\boldsymbol{r}}_{0}^{\mathcal{L}, 0}\right] \\
& =\boldsymbol{r}_{0}^{\mathcal{K}, \pm 1}, \\
\boldsymbol{r}_{0}^{\mathcal{K}, 0} & =\frac{1}{\sqrt{2}}\left[\boldsymbol{r}_{0}^{\mathcal{L}, 1}+\boldsymbol{r}_{0}^{\mathcal{L},-1}\right] \\
\Rightarrow e^{-\beta \overline{\mathcal{M}}} \boldsymbol{r}_{0}^{\mathcal{K}, 0} & =\frac{1}{\sqrt{2}}\left[e^{-\imath \beta} \boldsymbol{r}_{0}^{\mathcal{L}, 1}+e^{\imath \beta} \boldsymbol{r}_{0}^{\mathcal{L},-1}\right] \\
& =\frac{1}{2}\left[e^{-\imath \beta}\left(\imath \tilde{\boldsymbol{r}}_{0}^{\mathcal{K}, 0}+\boldsymbol{r}_{0}^{\mathcal{K}, 0}\right)+e^{\imath \beta}\left(-\imath \tilde{\boldsymbol{r}}_{0}^{\mathcal{K}, 0}+\boldsymbol{r}_{0}^{\mathcal{K}, 0}\right)\right] \\
& \sin (\beta) \tilde{\boldsymbol{r}}_{0}^{\mathcal{K}, 0}+\cos (\beta) \boldsymbol{r}_{0}^{\mathcal{K}, 0}, \\
\tilde{\boldsymbol{r}}_{0}^{\mathcal{K}, 0} & =\frac{1}{\sqrt{2}}\left[\imath \boldsymbol{r}_{0}^{\mathcal{L}, 1}-\imath \boldsymbol{r}_{0}^{\mathcal{L},-1}\right] \\
e^{-\beta \overline{\mathcal{M}}} \tilde{\boldsymbol{r}}_{0}^{\mathcal{K}, 0} & =\frac{\imath}{\sqrt{2}}\left[e^{-\imath \beta} \boldsymbol{r}_{0}^{\mathcal{L}, 1}-e^{\imath \beta} \boldsymbol{r}_{0}^{\mathcal{L},-1}\right] \\
& =\cos (\beta) \tilde{\boldsymbol{r}}_{0}^{\mathcal{K}, 0}+\sin (\beta) \boldsymbol{r}_{0}^{\mathcal{K}, 0} .
\end{aligned}
$$

It follows that we can simplify the expansion in $\mathcal{B}$ even more by noting that

$$
\begin{array}{r}
e^{-\beta \overline{\mathcal{M}}}\left[\sum_{k \neq 0, \gamma} \sigma_{k}^{\mathcal{K}, \gamma}(t) e^{-\imath s \omega_{k}^{\mathcal{K}, \gamma}} \boldsymbol{r}_{k}^{\mathcal{K}, \gamma}+\sum_{\gamma} \sigma_{0}^{\mathcal{K}, \gamma}(t) e^{-\imath s \omega_{0}^{\mathcal{K}, \gamma}} \boldsymbol{r}_{0}^{\mathcal{K}, \gamma}\right] \\
=\sum_{\{k \neq 0\} \cup\{\gamma \neq 0\}} \sigma_{k}^{\mathcal{K}, \gamma}(t) e^{-\imath s \omega_{k}^{\mathcal{K}, \gamma}} \boldsymbol{r}_{k}^{\mathcal{K}, \gamma}+\sigma_{0}^{\mathcal{K}, 0}(t)\left[\sin (\beta) \tilde{\boldsymbol{r}}_{0}^{\mathcal{K}, 0}+\cos (\beta) \boldsymbol{r}_{0}^{\mathcal{K}, 0}\right] \\
+\tilde{\sigma}_{0}^{\mathcal{K}, 0}(t)\left[\cos (\beta) \tilde{\boldsymbol{r}}_{0}^{\mathcal{K}, 0}+\sin (\beta) \boldsymbol{r}_{0}^{\mathcal{K}, 0}\right],
\end{array}
$$


which implies that the fast components of the flow can not influence the slow, i.e. since the operation $e^{-\beta \overline{\mathcal{M}}}$ does not affect any of the eigenvectors for $\gamma \neq 0$ then the impact of fast-fast waves onto slow reduces to that already computed in [43] where it was already shown (see Appendix B of [43]) that these interaction coefficients vanish identically. Thus, just as in the case of a single limit such as those studied in $[13,14,43]$, to $O(1)$ the fast waves cannot effect the evolution of the slow manifold.

\subsection{Strong stratification dominating rapid rotation}

Now we will investigate the limit of asymptotically strong stratification that is asymptotically stronger than the asymptotically rapid rotation. In this case we can directly apply the theory of Section 2 as the two linear operators have the appropriate label. We will expand the solution $\boldsymbol{u}$ as in (57) except in this Section, $\boldsymbol{r}_{k}^{\mathcal{L}, \gamma}$ are the eigenvectors of the fastest operator $\mathcal{L}$ as given in Section 5.3. The details are omitted in this case, as the calculations, while tedious, are straightforward and similar to the case considered above. We see that the linear operator $\overline{\mathcal{M}}$ can be interpreted as:

$$
\begin{aligned}
\overline{\mathcal{M}} \boldsymbol{u} & =e^{\imath \boldsymbol{k} \cdot \boldsymbol{x}} \sum_{\gamma, k}\left\langle\boldsymbol{u}, \boldsymbol{r}_{k}^{\gamma}\right\rangle \overline{\mathcal{M}} \boldsymbol{r}_{k}^{\gamma} \\
& =e^{\imath m z}\left[\imath\left\langle\boldsymbol{u}, \boldsymbol{r}_{m}^{1}\right\rangle \boldsymbol{r}_{m}^{1}-\imath\left\langle\boldsymbol{u}, \boldsymbol{r}_{m}^{-1}\right\rangle \boldsymbol{r}_{m}^{-1}\right]+\left\langle\boldsymbol{u}, \boldsymbol{r}_{0}^{0}\right\rangle \tilde{\boldsymbol{r}}_{0}^{0}+\left\langle\boldsymbol{u}, \tilde{\boldsymbol{r}}_{0}^{0}\right\rangle \boldsymbol{r}_{0}^{0},
\end{aligned}
$$

which can be rewritten as

$$
\overline{\mathcal{M}} \boldsymbol{u}=\left(\begin{array}{c}
-\langle v\rangle-\langle v\rangle_{H} \\
\langle u\rangle+\langle u\rangle_{H} \\
0 \\
0
\end{array}\right)
$$

where as before $\langle\cdot\rangle$ indicates the complete spatial average, and now $\langle\cdot\rangle_{H}$ indicates the horizontal spatial average. Thus, $\overline{\mathcal{M}}$ reduces to $\mathcal{K}$ on the horizontal and total mean $\left(|\boldsymbol{k}|=0\right.$ or $\left.\left|\boldsymbol{k}_{H}\right|=0\right)$ but vanishes otherwise $\left(\left|\boldsymbol{k}_{H}\right| \neq 0\right)$ indicating that the effect of asymptotically stronger stratification is to reduce the rotation to affect only the horizontal and total spatial average of the horizontal velocity.

This leads to the projection onto the null space of $\overline{\mathcal{M}}$ as

$$
P_{\overline{\mathcal{M}}} \boldsymbol{u}=\left(\begin{array}{c}
\boldsymbol{v}_{H}-\left\langle\boldsymbol{v}_{H}\right\rangle-\left\langle\boldsymbol{v}_{H}\right\rangle_{H} \\
0 \\
0
\end{array}\right) .
$$

Coupled with the action of $\mathcal{L}$ as the fastest linear operator, this leads to the evolution of the $O(1)$ slow dynamics:

$$
\begin{aligned}
\frac{\partial \boldsymbol{v}_{H}^{S}}{\partial t}+\boldsymbol{v}_{H}^{S} \cdot \nabla_{H} \boldsymbol{v}_{H}^{S}+\nabla_{H} \Delta_{H}^{-1} \nabla_{H} \cdot\left(\boldsymbol{v}_{H}^{S} \cdot \nabla_{H} \boldsymbol{v}_{H}^{S}\right) & =\frac{1}{\operatorname{Re}} \Delta \boldsymbol{v}_{H}^{S} \\
\nabla_{H} \cdot \boldsymbol{v}_{H}^{S} & =0 \\
\frac{\partial \rho^{S}}{\partial t} & =\frac{1}{\operatorname{Re} \operatorname{Pr}} \frac{\partial^{2} \rho^{S}}{\partial z^{2}}
\end{aligned}
$$


where

$$
\boldsymbol{v}_{H}^{S}=\boldsymbol{v}_{H}-\left\langle\boldsymbol{v}_{H}\right\rangle-\left\langle\boldsymbol{v}_{H}\right\rangle_{H} .
$$

The computation of the fast wave-slow dynamics interaction coefficients is more complicated in this case due to the action of $\overline{\mathcal{M}}$ on the horizontal as well as the total mean, but the final result is the same, there are no fast-fast-slow interactions meaning once again that to first order the fast waves cannot influence the evolution of the slow manifold.

\section{Conclusions and Discussion}

Using three different asymptotic methods, we have presented the derivation of a limiting system when there are two distinct fast temporal scales driven by skewHermitian linear operators with a discrete spectrum. The method of multiple scales indicates that the resultant $O(1)$ slow dynamics are dependent only on which scale is dominant, not on the actual relationship between the two small parameters. The specific example of the rotating, stratified Boussinesq equations indicates that the effect of the second fastest time scale is only seen in an averaged sense (either averaging across the entire domain or horizontally). As indicated by the theory of fast wave averaging (see Section 4) we have also shown explicitly that for the Boussinesq system there are no interactions between the two types of fast waves and the slow dynamics in the limiting system (up to $O(1)$ only).

The results presented in this paper have implications for designing the type of multi-scale parallel-in-time extended algorithm addressed by [20] because the current findings give mathematical insight for constructing a general parallel-intime coarse propagator where there is more than one frequency involved in creating the low frequency dynamics. In particular, (17) shows that construction of the frequency averaged nonlinear term, crucial for proving convergence of the parallelin-time algorithm in citeHaWi2013, includes two time averages, rather than one. We also describe the construction of a generalized averaged linear propagator given by (11) and (12) that is essential to the algorithm.

Finally, we discuss the limitations of our main results. That is, the applicability of the mathematical results may be questioned with regard to the effect of the next order terms in the asymptotic expansion, and as described in Section 4 the application of the rigorous theory of cancellation of oscillations does not generalize to the same level as the multiple scales calculation indicates. However, the absence of a fast-fast-slow wave interaction for each of these limiting systems in the Boussinesq equations does indicate some level of robustness to the result, regardless of the restrictions on the methodology and potential relationship between the two relevant small parameters. In essence, while it may be true that the reduced system derived here is valid only in a limited parameter regime due to the restrictions already described, it is almost certainly the case that the dynamics of the system in the presence of two fast, but distinctively different time scales is different from that when the two scales are equivalent. For the Boussinesq equations the rub of the matter is that quasi-geostrophy is not the only possible reduced system when the rotation and stratification are both strong, validating the computational investigations of [38] wherein it was observed that the concentration of energy is fundamentally different for Burger numbers distinct from unity. 
Acknowledgements We wish to thank the 2 anonymous referees whose comments significantly enhanced the presentation and scope of this article. J. P. W. would like to thank A. Larios, K. Julien, G. Chini, and A. Farhat for various discussions that prompted and motivated this work as well as generous support from the Mathematics Department of Brigham Young University. All of the authors wish to acknowledge the DOE LANL/LDRD program for support, as well as the hospitality of the Courant Institute of New York University where some of this work was completed. Wingate also wishes to thank the University of Exeter for support during the completion of this manuscript.

\section{A Commutation of the pertinent linear operators}

To make the fast-wave averaging equation computable and useful, we demonstrate explicitly that the linear operators $\mathcal{L}$ and $\overline{\mathcal{M}}$ commute, where $\mathcal{M}(\alpha)$ is defined as (12) and $\overline{\mathcal{M}}=$ $\lim _{\alpha \rightarrow \infty} \mathcal{M}(\alpha)$. First, suppose that the countable orthonormal eigenvectors of $\mathcal{L}$ are given by $\boldsymbol{r}_{k}$ with corresponding eigenvalues $\lambda_{k}$. Then for any vector $\boldsymbol{x}$

$$
\boldsymbol{x}=\sum_{k}\left\langle\boldsymbol{x}, \boldsymbol{r}_{k}\right\rangle \boldsymbol{r}_{k} \Rightarrow \mathcal{L} \boldsymbol{x}=\sum_{k} \lambda_{k}\left\langle\boldsymbol{x}, \boldsymbol{r}_{k}\right\rangle \boldsymbol{r}_{k}
$$

where as in the main body, $\langle\cdot, \cdot\rangle$ denotes the appropriate inner product. Further, the matrix exponential applied to $\boldsymbol{x}$ becomes

$$
e^{s \mathcal{L}} \boldsymbol{x}=\sum_{k}\left\langle\boldsymbol{x}, \boldsymbol{r}_{k}\right\rangle e^{s \lambda_{k}} \boldsymbol{r}_{k} \Rightarrow e^{s \mathcal{L}} \mathcal{K} e^{-s \mathcal{L}} \boldsymbol{x}=\sum_{k}\left\langle\boldsymbol{x}, \boldsymbol{r}_{k}\right\rangle \sum_{j}\left\langle\mathcal{K} \boldsymbol{r}_{k}, \boldsymbol{r}_{j}\right\rangle \boldsymbol{r}_{j} e^{s\left(\lambda_{j}-\lambda_{k}\right)} .
$$

Hence we see that

$$
\begin{aligned}
\mathcal{L} \mathcal{M}(\alpha) \boldsymbol{x} & =\mathcal{L}\left(\frac{1}{\alpha} \int_{0}^{\alpha} e^{s \mathcal{L}} \mathcal{K} e^{-s \mathcal{L}} d s\right) \boldsymbol{x} \\
& =\sum_{k, j} \lambda_{j}\left\langle\boldsymbol{x}, \boldsymbol{r}_{k}\right\rangle\left\langle\mathcal{K} \boldsymbol{r}_{k}, \boldsymbol{r}_{j}\right\rangle \boldsymbol{r}_{j}\left(\frac{1}{\alpha} \int_{0}^{\alpha} e^{s\left(\lambda_{j}-\lambda_{k}\right)} d s\right)
\end{aligned}
$$

whereas

$$
\begin{aligned}
\mathcal{M}(\alpha) \mathcal{L} \boldsymbol{x} & =\sum_{k}\left\langle\boldsymbol{x}, \boldsymbol{r}_{k}\right\rangle \lambda_{k} \mathcal{M}(\alpha) \boldsymbol{r}_{k} \\
& =\sum_{k, j} \lambda_{k}\left\langle\boldsymbol{x}, \boldsymbol{r}_{k}\right\rangle\left\langle\mathcal{K} \boldsymbol{r}_{k}, \boldsymbol{r}_{j}\right\rangle \boldsymbol{r}_{j}\left(\frac{1}{\alpha} \int_{0}^{\alpha} e^{s\left(\lambda_{j}-\lambda_{k}\right)} d s\right) .
\end{aligned}
$$

Note the subtle difference between (88) and (90), the sum is over a different eigenvalue: $\lambda_{j}$ for (88) and $\lambda_{k}$ for (90). This implies that $\mathcal{L}$ and $\mathcal{M}(\alpha)$ do not commute, however if we consider the limit of $\alpha \rightarrow \infty$ then note that because $\mathcal{L}$ is skew-Hermitian (implying all of the $\lambda_{k}$ are pure imaginary or vanishing) then

$$
\lim _{\alpha \rightarrow \infty} \frac{1}{\alpha} \int_{0}^{\alpha} e^{s\left(\lambda_{j}-\lambda_{k}\right)} d s=\left\{\begin{array}{ll}
1 & \text { if } \lambda_{k}=\lambda_{j} \\
0 & \text { if } \lambda_{k} \neq \lambda_{j}
\end{array} .\right.
$$

It follows directly that $\overline{\mathcal{M}} \mathcal{L} \boldsymbol{x}=\mathcal{L} \overline{\mathcal{M}} \boldsymbol{x}$, and hence the long time limiting linear operator $\overline{\mathcal{M}}$ does commute with $\mathcal{L}$.

\section{References}

1. G. Ariel, B. Engquist, S. J. Kim, and R. Tsai. Iterated averaging of three-scale oscillatory systems. Technical Report ICES REPORT 13-01, The Institute for Computational Enginerring and Sciences, The University of Texas, 2013. 
2. G. Ariel, B. Engquist, and R. Tsai. A multi scale method for highly oscillatory ordinary differential equations with resonance. Mathematics of Computation, 78(266):929-956, 2009.

3. N. N. Bogoliubov and Y. A. Mitropolsky. Asymptotic Methods in the Theory of Non-linear Oscillations. Gordon and Breach, 1961.

4. A. Bourgeois and J. T. Beale. Validity of the quasigeostrophic model for large scale flow in the atmosphere and ocean. SIAM Journal of Mathematical Analysis, 25:1023-1068, 1994.

5. D. Bresch, D. Gérard-Varet, and E. Grenier. Derivation of the planetary geostrophic equations. Archives of Rational Mechanics and Analysis, 182:387-413, 2006.

6. J. G. Charney. On the scale of atmospheric motions. Geophysical Publications, 17(2):17 pages, 1948.

7. J. G. Charney. On a physical basis for numerical prediction of large-scale motions in the atmosphere. Journal of Meteorology, 6(6):371-385, 1949.

8. J. G. Charney, R. Fjortoft, and J. von Neumann. Numerical integration of the barotropic vorticity equation. Tellus, 2:237-254, 1950.

9. J. Y. Chemin. A propos d'un probléme de pénalisation de type anti symétrique. Comptes Rendus de l'Académie des Sceinces - Series I, 321(7):861-864, 1995.

10. Lin-Yuan Chen, Nigel Goldenfeld, and Y. Oono. Erratum: "Renormalization group theory for global asymptotic analysis" [Phy. Rev. Lett. 73 (1994), no. 10, 1311-1315; MR1289625 (95d:81089)]. Phys. Rev. Lett., 74(10):1889, 1995.

11. Lin-Yuan Chen, Nigel Goldenfeld, and Y. Oono. The Renormalization group and singular perturbations: Multiple scales, boundary layers and reductive perturbation theory. Phys.Rev., E54:376-394, 1996.

12. C. Cheverry. Propagation of Oscillations in Real Vanishing Viscosity Limit. Communications in Mathematical Physics, 247:655-695, 2004.

13. P. F. Embid and A. J. Majda. Averaging over fast gravity waves for geophysical flows with arbitrary potential vorticity. Communications in Partial Differential Equations, 21(3-4):619-658, 1996.

14. P. F. Embid and A. J. Majda. Low Froude number limiting dynamics for stably stratified flow with small or finite Rossby numbers. Geophysical and Astrophysical Fluid Dynamics, 87(1-2):1-50, 1998.

15. B. Engquist and R. Tsai. Heterogeneous multi scale methods for stiff ordinary differential equations. Mathematics of Computation, 74(252):1707-1742, 2005.

16. E. Feireisl and A. Novotny. Multiple Scales and Singular Limits for Compressible Rotating Fluids with General Initial Data. Communications in Partial Differential Equations, 39:1104-1127, 2014.

17. M. J. Gander. 50 years of time parallel time integration. In T. Carraro, M. Geiger, S. Korkel, and R. Rannacher, editors, Multiple Shooting and Time Domain Decomposition. Springer-Verlag, 2015.

18. Martin J. Gander and Ernst Hairer. Analysis for parareal algorithms applied to hamiltonian differential equations. J. Comput. Appl. Math., 259:2-13, March 2014.

19. E. Grenier. Oscillatory perturbations of the navier stokes equations. Journal de Matheématiques Pures et Appliquées, 76(6):477-498, 1997.

20. T. Haut and B. A. Wingate. An asymptotic parallel-in-time method for highly oscillatory pdes. SIAM Journal of Numerical Analysis, submitted, 2013.

21. Darryl D. Holm and Vladimir Zeitlin. Hamilton's princple for quasigeostrophic motion. Phyiscs of Fluids, 10:800-806, 1998.

22. K. Julien, E. Knobloch, R. Milliff, and J. Werne. Generalized Quasi-Geostrophy for spatially anisotropic rotationally constrained flows. Journal of Fluid Mechanics, 555:233-274, 2006.

23. S. Klainerman and A. J. Majda. Singular limits of quasilinear hyperbolic systems with large parameters and the incompressible limit of compressible fluids. Communications in Pure and Applied Mathematics, 34(4):481-524, 1981.

24. P. Lelong and J. J. Riley. Internal wave-vortical mode interactions in strongly stratified flows. Journal of Fluid Mechanics, 232:1-19, 1991.

25. J Lions, Yvon Maday, and Gabriel Turinici. A"parareal"in time discretization of pde's. Comptes Rendus de l'Academie des Sciences Series I Mathematics, 332(7):661-668, 2001.

26. E. N Lorenz. Attractor Sets and Quasi-Geostrophic Equilibrium. Journal of the Atmospheric Sciences, 37:1685-1699, 1980.

27. A. J. Majda and M. J. Grote. Model dynamics and vertical collapse in decaying strongly stratified flows. Physics of fluids, 9(10):2932-2940, 1997. 
28. I. Moise and M. Ziane. Renormalization group method. Applications to partial differential equations. J. Dynam. Differential Equations, 13(2):275-321, 2001.

29. D. J. Muraki, C. Snyder, and R. Rotunno. The next-order corrections to quasigeostrophic theory. Journal of the Atmospheric Sciences, 56:1547-1560, 1999.

30. J. Pedlosky. Geophysical Fluid Dynamics. Springer-Verlag, 1987.

31. M. Petcu, R. M. Temam, and D. Wirosoetisno. Renormalization group method applied to the primitive equations. Journal of Differential Equations, 208:215-257, 2005.

32. M. Remmel and L. Smith. New intermediate models for rotating shallow water and an investigation of the preference for anticyclones. Journal of Fluid Mechanics, 635:321-359, 2009.

33. J. J. Riley and S. M. DeBruynKops. Dynamics of turbulence strongly influenced by buoyancy. Physics of Fluids, 15(7):2047-2059, 2003.

34. S. Schochet. Fast singular limits of hyperbolic pde's. Journal of Differential Equations, 114:476-512, 1994.

35. S. Schochet. The Mathematical Theory of Low Mach Number Flows. ESAIM: Mathematical Modelling and Numerical Analysis, 39(3):441-458, 2005.

36. L. M. Smith and F. Waleffe. Transfer of energy to two-dimensional large scales in forced, rotating three-dimensional turbulence. Physics of Fluids, 11(6):1608-1622, 1999.

37. L. M. Smith and F. Waleffe. Generation of slow large scales in forced rotating stratified turbulence. Journal of Fluid Mechanics, 451:145-168, 2002.

38. Jai Sukhatme and Leslie M Smith. Vortical and wave modes in 3d rotating stratified flows: random large-scale forcing. Geophysical and Astrophysical Fluid Dynamics, 102(5):437455, 2008.

39. R. M. Temam and D. Wirosoetisno. On the solutions of the renormalized equations at all orders. Advances in Differential Equations, 8(8):1005-1024, 2003.

40. R. M. Temam and D. Wirosoetisno. Exponential approximations for the primitive equations of the ocean. Discrete and continuous dynamical systems: series B, 7(2):425-440, 2007.

41. R. M. Temam and D. Wirosoetisno. Stability of the slow manifold in the primitive equations. SIAM Journal of Mathematical Analysis, 42(1):427-458, 2010.

42. T. Warn, O. Bokhove, T. G. Shepherd, and G. K. Vallis. Rossby number expansions, slaving principles, and balance dynamics. Quarterly Journal of the Royal Meteorological Society, 121:723-739, 1995.

43. B. A. Wingate, P. Embid, M. Holmes-Cerfon, and M. A. Taylor. Low Rossby limiting dynamics for stably stratified flow with finite Froude number. Journal of Fluid Mechanics, 676:546-571, 2011.

44. D. Wirosoetisno, T. G. Shepherd, and R. M. Temam. Free Gravity Waves and Balanced Dynamics. Journal of the Atmospheric Sciences, 59(23):3382-3398, 2002. 